\title{
Iterative Algorithms for Solving the System of Mixed Equilibrium Problems, Fixed-Point Problems, and Variational Inclusions with Application to Minimization Problem
}

\author{
Tanom Chamnarnpan and Poom Kumam
}

Department of Mathematics, Faculty of Science, King Mongkut's University of Technology Thonburi (KMUTT), Bangmod, Thrungkru, Bangkok 10140, Thailand

Correspondence should be addressed to Poom Kumam, poom.kum@kmutt.ac.th

Received 7 October 2011; Accepted 1 November 2011

Academic Editor: Yeong-Cheng Liou

Copyright (C) 2012 T. Chamnarnpan and P. Kumam. This is an open access article distributed under the Creative Commons Attribution License, which permits unrestricted use, distribution, and reproduction in any medium, provided the original work is properly cited.

We introduce a new iterative algorithm for solving a common solution of the set of solutions of fixed point for an infinite family of nonexpansive mappings, the set of solution of a system of mixed equilibrium problems, and the set of solutions of the variational inclusion for a $\beta$-inversestrongly monotone mapping in a real Hilbert space. We prove that the sequence converges strongly to a common element of the above three sets under some mild conditions. Furthermore, we give a numerical example which supports our main theorem in the last part.

\section{Introduction}

Let $C$ be a closed convex subset of a real Hilbert space $H$ with the inner product $\langle\cdot, \cdot\rangle$ and the norm $\|\cdot\|$. Let $F$ be a bifunction of $C \times C$ into $R$, where $R$ is the set of real numbers, $\varphi: C \rightarrow R$ be a real-valued function. Let $\Lambda$ be arbitrary index set. The system of mixed equilibrium problem is for finding $x \in C$ such that

$$
F_{k}(x, y)+\varphi(y)-\varphi(x) \geq 0, \quad k \in \Lambda, \forall y \in C
$$

The set of solutions of (1.1) is denoted by $\operatorname{SMEP}\left(F_{k}\right)$, that is,

$$
\operatorname{SMEP}\left(F_{k}\right)=\left\{x \in C:=F_{k}(x, y)+\varphi(y)-\varphi(x) \geq 0, k \in \Lambda, \forall y \in C\right\} .
$$


If $\Lambda$ is a singleton, then problem (1.1) becomes the following mixed equilibrium problem: finding $x \in C$ such that

$$
F(x, y)+\varphi(y)-\varphi(x) \geq 0, \quad \forall y \in C
$$

The set of solutions of (1.3) is denoted by $\operatorname{MEP}(F)$. such that

If $\varphi \equiv 0$, the problem (1.3) is reduced into the equilibrium problem [1] for finding $x \in C$

$$
F(x, y) \geq 0, \quad \forall y \in C
$$

The set of solutions of (1.4) is denoted by $\mathrm{EP}(F)$. This problem contains fixed-point problems, includes as special cases numerous problems in physics, optimization, and economics. Some methods have been proposed to solve the system of mixed equilibrium problem and the equilibrium problem, please consult [2-19].

Recall that, a mapping $S: C \rightarrow C$ is said to be nonexpansive if

$$
\|S x-S y\| \leq\|x-y\|,
$$

for all $x, y \in C$. If $C$ is a bounded closed convex and $S$ is a nonexpansive mapping of $C$ into itself, then $F(S)$ is nonempty [20]. Let $A: C \rightarrow H$ be a mapping, the Hartmann-Stampacchia variational inequality for finding $x \in C$ such that

$$
\langle A x, y-x\rangle \geq 0, \quad \forall y \in C \text {. }
$$

The set of solutions of (1.6) is denoted by $\operatorname{VI}(C, A)$. The variational inequality has been extensively studied in the literature [21-28].

Iterative methods for nonexpansive mappings have recently been applied to solve convex minimization problems. Convex minimization problems have a great impact and influence on the development of almost all branches of pure and applied sciences. A typical problem is to minimize a quadratic function over the set of the fixed points of a nonexpansive mapping on a real Hilbert space $H$ :

$$
\theta(x)=\frac{1}{2}\langle A x, x\rangle-\langle x, y\rangle, \quad \forall x \in F(S),
$$

where $A$ is a linear bounded operator, $F(S)$ is the fixed point set of a nonexpansive mapping $S$, and $y$ is a given point in $H$ [29].

We denote weak convergence and strong convergence by notations $\rightarrow$ and $\rightarrow$, respectively. A mapping $A$ of $C$ into $H$ is called monotone if

$$
\langle A x-A y, x-y\rangle \geq 0,
$$


for all $x, y \in C$. A mapping $A$ of $C$ into $H$ is called $\alpha$-inverse-strongly monotone if there exists a positive real number $\alpha$ such that

$$
\langle A x-A y, x-y\rangle \geq \alpha\|A x-A y\|^{2}
$$

for all $x, y \in C$. It is obvious that any $\alpha$-inverse-strongly monotone mappings $A$ are monotone and Lipschitz continuous mapping. A linear bounded operator $A$ is strongly positive if there exists a constant $\bar{\gamma}>0$ with the property

$$
\langle A x, x\rangle \geq \bar{\gamma}\|x\|^{2}
$$

for all $x \in H$. A self-mapping $f: C \rightarrow C$ is a contraction on $C$ if there exists a constant $\alpha \in(0,1)$ such that

$$
\|f(x)-f(y)\| \leq \alpha\|x-y\|
$$

for all $x, y \in C$. We use $\Pi_{C}$ to denote the collection of all contraction on $C$. Note that each $f \in \Pi_{C}$ has a unique fixed point in $C$.

Let $B: H \rightarrow H$ be a single-valued nonlinear mapping and $M: H \rightarrow 2^{H}$ be a setvalued mapping. The variational inclusion problem is to find $x \in H$ such that

$$
\theta \in B(x)+M(x)
$$

where $\theta$ is the zero vector in $H$. The set of solutions of problem (1.12) is denoted by $I(B, M)$. The variational inclusion has been extensively studied in the literature, see, for example, [3032] and the reference therein.

A set-valued mapping $M: H \rightarrow 2^{H}$ is called monotone if for all $x, y \in H, f \in M(x)$, and $g \in M(y)$ impling $\langle x-y, f-g\rangle \geq 0$. A monotone mapping $M$ is maximal if its graph $G(M):=\{(f, x) \in H \times H: f \in M(x)\}$ of $M$ is not properly contained in the graph of any other monotone mapping. It is known that a monotone mapping $M$ is maximal if and only if for $(x, f) \in H \times H,\langle x-y, f-g\rangle \geq 0$ for all $(y, g) \in G(M)$ impling $f \in M(x)$.

Let $B$ be an inverse-strongly monotone mapping of $C$ into $H$, and let $N_{C} v$ be normal cone to $C$ at $v \in C$, that is, $N_{C} v=\{w \in H:\langle v-u, w\rangle \geq 0$, for all $u \in C\}$, and define

$$
T v= \begin{cases}B v+N_{C} v, & \text { if } v \in C, \\ \emptyset, & \text { if } v \notin C .\end{cases}
$$

Then, $T$ is a maximal monotone and $\theta \in T v$ if and only if $v \in \operatorname{VI}(C, B)$ (see [33]).

Let $M: H \rightarrow 2^{H}$ be a set-valued maximal monotone mapping, then the single-valued mapping $J_{M, \lambda}: H \rightarrow H$ defined by

$$
J_{M, \lambda}(x)=(I+\lambda M)^{-1}(x), \quad x \in H,
$$

is called the resolvent operator associated with $M$, where $\lambda$ is any positive number and $I$ is the identity mapping. It is worth mentioning that the resolvent operator is nonexpansive, 
1-inverse-strongly monotone, and that a solution of problem (1.12) is a fixed point of the operator $J_{M, \lambda}(I-\lambda B)$ for all $\lambda>0$, (for more details see [34]).

In 2000, Moudafi [35] introduced the viscosity approximation method for nonexpansive mappings and proved that if $H$ is a real Hilbert space, the sequence $\left\{x_{n}\right\}$ defined by the iterative method below, with the initial guess $x_{0} \in C$ is chosen arbitrarily,

$$
x_{n+1}=\alpha_{n} f\left(x_{n}\right)+\left(1-\alpha_{n}\right) S x_{n}, \quad n \geq 0,
$$

where $\left\{\alpha_{n}\right\} \subset(0,1)$ satisfies certain conditions and converges strongly to a fixed point of $S$ (say $\bar{x} \in C$ ), which is then a unique solution of the following variational inequality:

$$
\langle(I-f) \bar{x}, x-\bar{x}\rangle \geq 0, \quad \forall x \in F(S)
$$

In 2006, Marino and $\mathrm{Xu}$ [29] introduced a general iterative method for nonexpansive mapping. They defined the sequence $\left\{x_{n}\right\}$ generated by the algorithm $x_{0} \in C$,

$$
x_{n+1}=\alpha_{n} \gamma f\left(x_{n}\right)+\left(I-\alpha_{n} A\right) S x_{n}, \quad n \geq 0,
$$

where $\left\{\alpha_{n}\right\} \subset(0,1)$, and $A$ is a strongly positive linear bounded operator. They proved that if $C=H$, and the sequence $\left\{\alpha_{n}\right\}$ satisfies appropriate conditions, then the sequence $\left\{x_{n}\right\}$ generated by (1.17) converges strongly to a fixed point of $S$ (say $\bar{x} \in H$ ) which is the unique solution of the following variational inequality:

$$
\langle(A-\gamma f) \bar{x}, x-\bar{x}\rangle \geq 0, \quad \forall x \in F(S)
$$

which is the optimality condition for the minimization problem

$$
\min _{x \in F(S) \cap \mathrm{EP}(F)} \frac{1}{2}\langle A x, x\rangle-h(x)
$$

where $h$ is a potential function for $\gamma f$ (i.e., $h^{\prime}(x)=\gamma f(x)$ for $x \in H$ ).

For finding a common element of the set of fixed points of nonexpansive mappings and the set of solution of the variational inequalities. Let $P_{C}$ be the projection of $H$ onto $C$. In 2005, Iiduka and Takahashi [36] introduced the following iterative process for $x_{0} \in C$,

$$
x_{n+1}=\alpha_{n} u+\left(1-\alpha_{n}\right) S P_{C}\left(x_{n}-\lambda_{n} A x_{n}\right), \quad \forall n \geq 0,
$$

where $u \in C,\left\{\alpha_{n}\right\} \subset(0,1)$, and $\left\{\lambda_{n}\right\} \subset[a, b]$ for some $a, b$ with $0<a<b<2 \beta$. They proved that under certain appropriate conditions imposed on $\left\{\alpha_{n}\right\}$ and $\left\{\lambda_{n}\right\}$, the sequence $\left\{x_{n}\right\}$ generated by (1.20) converges strongly to a common element of the set of fixed points of a nonexpansive mapping and the set of solutions of the variational inequality for an inversestrongly monotone mapping (

$$
\langle\bar{x}-u, x-\bar{x}\rangle \geq 0, \quad \forall x \in F(S) \cap \operatorname{VI}(C, \mathrm{~A}) .
$$


In 2008, Su et al. [37] introduced the following iterative scheme by the viscosity approximation method in a real Hilbert space: $x_{1}, u_{n} \in H$

$$
\begin{gathered}
F\left(u_{n}, y\right)+\frac{1}{r_{n}}\left\langle y-u_{n}, u_{n}-x_{n}\right\rangle \geq 0, \quad \forall y \in C, \\
x_{n+1}=\alpha_{n} f\left(x_{n}\right)+\left(1-\alpha_{n}\right) S P_{C}\left(u_{n}-\lambda_{n} A u_{n}\right),
\end{gathered}
$$

for all $n \in \mathbb{N}$, where $\left\{\alpha_{n}\right\} \subset[0,1)$ and $\left\{r_{n}\right\} \subset(0, \infty)$ satisfing some appropriate conditions. Furthermore, they proved that $\left\{x_{n}\right\}$ and $\left\{u_{n}\right\}$ converge strongly to the same point $z \in F(S) \cap$ $\mathrm{VI}(C, A) \cap \operatorname{EP}(F)$, where $z=P_{F(S) \cap V I(C, A) \cap \operatorname{EP}(F)} f(z)$.

Let $\left\{T_{i}\right\}$ be an infinite family of nonexpansive mappings of $H$ into itself, and let $\left\{\lambda_{i}\right\}$ be a real sequence such that $0 \leq \lambda_{i} \leq 1$ for every $i \in N$. For $n \geq 1$, we defined a mapping $W_{n}$ of $H$ into itself as follows:

$$
\begin{aligned}
U_{n, n+1} & :=I, \\
U_{n, n} & :=\lambda_{n} T_{n} U_{n, n+1}+\left(1-\lambda_{n}\right) I, \\
& \vdots \\
U_{n, k} & :=\lambda_{k} T_{k} U_{n, k+1}+\left(1-\lambda_{k}\right) I, \\
& \vdots \\
U_{n, 2} & :=\lambda_{2} T_{2} U_{n, 3}+\left(1-\lambda_{2}\right) I, \\
W_{n} & :=U_{n, 1}:=\lambda_{1} T_{1} U_{n, 2}+\left(1-\lambda_{1}\right) I .
\end{aligned}
$$

In 2011, He et al. [38] introduced the following iterative process for $\left\{T_{n}: C \rightarrow C\right\}$ which is a sequence of nonexpansive mappings. Let $\left\{z_{n}\right\}$ be the sequence defined by

$$
z_{n+1}=\epsilon_{n} \gamma f\left(z_{n}\right)+\left(I-\epsilon_{n}\right) W_{n} K_{r_{1}, n}^{1} K_{r_{2}, n}^{2} \cdots K_{r_{K}, n}^{K} z_{n}, \quad \forall n \in N
$$

The sequence $\left\{z_{n}\right\}$ defined by (1.24) converges strongly to a common element of the set of fixed points of nonexpansive mappings, the set of solutions of the variational inequality, and the generalized equilibrium problem. Recently, Jitpeera and Kumam [39] introduced the following new general iterative method for finding a common element of the set of solutions of fixed point for nonexpansive mappings, the set of solution of generalized mixed equilibrium problems, and the set of solutions of the variational inclusion for a $\beta$-inversestrongly monotone mapping in a real Hilbert space.

In this paper, we modify the iterative methods (1.17), (1.22), and (1.24) by purposing the following new general viscosity iterative method: $x_{0}, u_{n} \in C$,

$$
\begin{gathered}
u_{n}=K_{r_{n}, n}^{F_{N}} \cdot K_{r_{n-1}, n}^{F_{N-1}} \cdot K_{r_{n-2}, n}^{F_{N-2}} \cdots \cdots K_{r_{2}, n}^{F_{2}} \cdot K_{r_{1}, n}^{F_{1}} \cdot x_{n}, \quad \forall n \in N \\
x_{n+1}=P_{C}\left[\epsilon_{n} \gamma f\left(x_{n}\right)+\left(I-\epsilon_{n} A\right) W_{n} J_{M, \lambda}\left(u_{n}-\lambda B u_{n}\right)\right],
\end{gathered}
$$


for all $n \in \mathbb{N}$, where $\left\{\alpha_{n}\right\} \subset(0,1),\left\{r_{n}\right\} \subset(0,2 \sigma)$, and $\lambda \in(0,2 \beta)$ satisfy some appropriate conditions. The purpose of this paper shows that under some control conditions the sequence $\left\{x_{n}\right\}$ converges strongly to a common element of the set of common fixed points of nonexpansive mappings, the solution of the system of mixed equilibrium problems, and the set of solutions of the variational inclusion in a real Hilbert space. Moreover, we apply our results to the class of strictly pseudocontractive mappings. Finally, we give a numerical example which supports our main theorem in the last part. Our results improve and extend the corresponding results of Marino and Xu [29], Su et al. [37], He et al. [38], and some authors.

\section{Preliminaries}

Let $H$ be a real Hilbert space and $C$ be a nonempty closed and convex subset of $H$. Recall that the (nearest point) projection $P_{C}$ from $H$ onto $C$ assigns to each $x \in H$ and the unique point in $P_{C} x \in C$ satisfies the property

$$
\left\|x-P_{C} x\right\|=\min _{y \in C}\|x-y\|
$$

which is equivalent to the following inequality

$$
\left\langle x-P_{C} x, P_{C} x-y\right\rangle \geq 0, \quad \forall y \in C
$$

The following characterizes the projection $P_{C}$. We recall some lemmas which will be needed in the rest of this paper.

Lemma 2.1. The function $u \in C$ is a solution of the variational inequality if and only if $u \in C$ satisfies the relation $u=P_{C}(u-\lambda B u)$ for all $\lambda>0$.

Lemma 2.2. For a given $z \in H, u \in C, u=P_{C} z \Leftrightarrow\langle u-z, v-u\rangle \geq 0, \forall v \in C$.

It is well known that $P_{C}$ is a firmly nonexpansive mapping of $H$ onto $C$ and satisfies

$$
\left\|P_{C} x-P_{C} y\right\|^{2} \leq\left\langle P_{C} x-P_{C} y, x-y\right\rangle, \quad \forall x, y \in H
$$

Moreover, $P_{C} x$ is characterized by the following properties: $P_{C} x \in C$ and for all $x \in H, y \in C$,

$$
\left\langle x-P_{C} x, y-P_{C} x\right\rangle \leq 0
$$

Lemma 2.3 (see [40]). Let $M: H \rightarrow 2^{H}$ be a maximal monotone mapping, and let $B: H \rightarrow H$ be a monotone and Lipshitz continuous mapping. Then the mapping $L=M+B: H \rightarrow 2^{H}$ is a maximal monotone mapping.

Lemma 2.4 (see [41]). Each Hilbert space $H$ satisfies Opial's condition, that is, for any sequence $\left\{x_{n}\right\} \subset H$ with $x_{n} \rightarrow x$, the inequality $\liminf _{n \rightarrow \infty}\left\|x_{n}-x\right\|<\liminf _{n \rightarrow \infty}\left\|x_{n}-y\right\|$, hold for each $y \in H$ with $y \neq x$. 
Lemma 2.5 (see [42]). Assume $\left\{a_{n}\right\}$ is a sequence of nonnegative real numbers such that

$$
a_{n+1} \leq\left(1-\gamma_{n}\right) a_{n}+\delta_{n}, \quad \forall n \geq 0,
$$

where $\left\{\gamma_{n}\right\} \subset(0,1)$ and $\left\{\delta_{n}\right\}$ is a sequence in $R$ such that

(i) $\sum_{n=1}^{\infty} \gamma_{n}=\infty$,

(ii) $\lim \sup _{n \rightarrow \infty} \delta_{n} / \gamma_{n} \leq 0$ or $\sum_{n=1}^{\infty}\left|\delta_{n}\right|<\infty$.

Then $\lim _{n \rightarrow \infty} a_{n}=0$.

Lemma 2.6 (see [43]). Let $C$ be a closed convex subset of a real Hilbert space $H$, and let $T: C \rightarrow C$ be a nonexpansive mapping. Then $I-T$ is demiclosed at zero, that is,

$$
x_{n} \longrightarrow x, \quad x_{n}-T x_{n} \longrightarrow 0,
$$

implying $x=T x$.

For solving the mixed equilibrium problem, let us assume that the bifunction $F: C \times$ $C \rightarrow \mathcal{R}$ and the nonlinear mapping $\varphi: C \rightarrow \mathcal{R}$ satisfy the following conditions:

(A1) $F(x, x)=0$ for all $x \in C$;

(A2) $F$ is monotone, that is, $F(x, y)+F(y, x) \leq 0$ for any $x, y \in C$;

(A3) for each fixed $y \in C, x \mapsto F(x, y)$ is weakly upper semicontinuous;

(A4) for each fixed $x \in C, y \mapsto F(x, y)$ is convex and lower semicontinuous;

(B1) for each $x \in C$ and $r>0$, there exist a bounded subset $D_{x} \subseteq C$ and $y_{x} \in C$ such that for any $z \in C \backslash D_{x}$,

$$
F\left(z, y_{x}\right)+\varphi\left(y_{x}\right)-\varphi(z)+\frac{1}{r}\left\langle y_{x}-z, z-x\right\rangle<0,
$$

(B2) $C$ is a bounded set.

Lemma 2.7 (see [44]). Let $C$ be a nonempty closed and convex subset of a real Hilbert space $H$. Let $F: C \times C \rightarrow R$ be a bifunction mapping satisfying (A1)-(A4), and let $\varphi: C \rightarrow R$ be a convex and lower semicontinuous function such that $C \cap \operatorname{dom} \varphi \neq \emptyset$. Assume that either (B1) or (B2) holds. For $r>0$ and $x \in H$, then there exists $u \in C$ such that

$$
F(u, y)+\varphi(y)-\varphi(u)+\frac{1}{r}\langle y-u, u-x\rangle \geq 0 .
$$

Define a mapping $K_{r}: H \rightarrow C$ as follows:

$$
K_{r}(x)=\left\{u \in C: F(u, y)+\varphi(y)-\varphi(u)+\frac{1}{r}\langle y-u, u-x\rangle \geq 0, \forall y \in C\right\},
$$


for all $x \in H$. Then, the following hold:

(i) $K_{r}$ is single-valued;

(ii) $K_{r}$ is firmly nonexpansive, that is, for any $x, y \in H,\left\|K_{r} x-K_{r} y\right\|^{2} \leq\left\langle K_{r} x-K_{r} y, x-y\right\rangle$;

(iii) $F\left(K_{r}\right)=\operatorname{MEP}(F)$;

(iv) $\operatorname{MEP}(F)$ is closed and convex.

Lemma 2.8 (see [29]). Assume $A$ is a strongly positive linear bounded operator on a Hilbert space $H$ with coefficient $\bar{\gamma}>0$ and $0<\rho \leq\|A\|^{-1}$, then $\|I-\rho A\| \leq 1-\rho \bar{\gamma}$.

Lemma 2.9 (see [38]). Let $C$ be a nonempty closed and convex subset of a strictly convex Banach space. Let $\left\{T_{i}\right\}_{i \in N}$ be an infinite family of nonexpansive mappings of $C$ into itself such that $\cap_{i \in N} F\left(T_{i}\right) \neq \emptyset$, and let $\left\{\lambda_{i}\right\}$ be a real sequence such that $0 \leq \lambda_{i} \leq b<1$ for every $i \in N$. Then $F(W)=\cap_{i \in N} F\left(T_{i}\right) \neq \emptyset$.

Lemma 2.10 (see [38]). Let $C$ be a nonempty closed and convex subset of a strictly convex Banach space. Let $\left\{T_{i}\right\}$ be an infinite family of nonexpansive mappings of $C$ into itself, and let $\left\{\lambda_{i}\right\}$ be a real sequence such that $0 \leq \lambda_{i} \leq b<1$ for every $i \in N$. Then, for every $x \in C$ and $k \in N$, the limit $\lim _{n \rightarrow \infty} U_{n, k}$ exist.

In view of the previous lemma, we define

$$
W x:=\lim _{n \rightarrow \infty} U_{n, 1} x=\lim _{n \rightarrow \infty} W_{n} x .
$$

\section{Strong Convergence Theorems}

In this section, we show a strong convergence theorem which solves the problem of finding a common element of the common fixed points, the common solution of a system of mixed equilibrium problems and variational inclusion of inverse-strongly monotone mappings in a Hilbert space.

Theorem 3.1. Let $H$ be a real Hilbert space and $C$ a nonempty close and convex subset of $H$, and let $B$ be a $\beta$-inverse-strongly monotone mapping. Let $\varphi: C \rightarrow R$ be a convex and lower semicontinuous function, $f: C \rightarrow C$ a contraction mapping with coefficient $\alpha(0<\alpha<1)$, and $M: H \rightarrow 2^{H} a$ maximal monotone mapping. Let $A$ be a strongly positive linear bounded operator of $H$ into itself with coefficient $\bar{\gamma}>0$. Assume that $0<\gamma<\bar{\gamma} / \alpha$ and $\lambda \in(0,2 \beta)$. Let $\left\{T_{n}\right\}$ be a family of nonexpansive mappings of $H$ into itself such that

$$
\theta:=\bigcap_{n=1}^{\infty} F\left(T_{n}\right) \cap\left(\bigcap_{k=1}^{N} \operatorname{SMEP}\left(F_{k}\right)\right) \cap I(B, M) \neq \emptyset .
$$

Suppose that $\left\{x_{n}\right\}$ is a sequence generated by the following algorithm for $x_{0} \in C$ arbitrarily and

$$
\begin{gathered}
u_{n}=K_{r_{n}, n}^{F_{N}} \cdot K_{r_{n-1}, n}^{F_{N-1}} \cdot K_{r_{n-2}, n}^{F_{N-2}} \cdots \cdots K_{r_{2}, n}^{F_{2}} \cdot K_{r_{1}, n}^{F_{1}} \cdot x_{n}, \quad \forall n \in N \\
x_{n+1}=P_{C}\left[\epsilon_{n} \gamma f\left(x_{n}\right)+\left(I-\epsilon_{n} A\right) W_{n} J_{M, \lambda}\left(u_{n}-\lambda B u_{n}\right)\right],
\end{gathered}
$$


for all $n=1,2,3, \ldots$, where

$$
\begin{array}{r}
K_{r_{i, n}}^{F_{i}}(x)=\left\{u_{n} \in C: F_{i}\left(u_{n}, y\right)+\varphi(y)-\varphi\left(u_{n}\right)+\frac{1}{r_{i, n}}\left\langle y-u_{n}, u_{n}-x_{n}\right\rangle \geq 0, \forall y \in C\right\} \\
i=1,2,3, \ldots, N
\end{array}
$$

and the following conditions are satisfied

(C1): $\left\{\epsilon_{n}\right\} \subset(0,1), \lim _{n \rightarrow 0} \epsilon_{n}=0, \sum_{n=1}^{\infty} \epsilon_{n}=\infty, \sum_{n=1}^{\infty}\left|\epsilon_{n+1}-\epsilon_{n}\right|<\infty$;

(C2): $\left\{r_{n}\right\} \subset[c, d]$ with $c, d \in(0,2 \sigma)$ and $\sum_{n=1}^{\infty}\left|r_{n+1}-r_{n}\right|<\infty$.

Then, the sequence $\left\{x_{n}\right\}$ converges strongly to $q \in \theta$, where $q=P_{\theta}(\gamma f+I-A)(q)$ which solves the following variational inequality:

$$
\langle(\gamma f-A) q, p-q\rangle \leq 0, \quad \forall p \in \theta,
$$

which is the optimality condition for the minimization problem

$$
\min _{q \in \theta} \frac{1}{2}\langle A q, q\rangle-h(q)
$$

where $h$ is a potential function for $\gamma f$ (i.e., $h^{\prime}(q)=\gamma f(q)$ for $\left.q \in H\right)$.

Proof. For condition (C1), we may assume without loss of generality, and $\epsilon_{n} \in\left(0,\|A\|^{-1}\right)$ for all $n$. By Lemma 2.8, we have $\left\|I-\epsilon_{n} A\right\| \leq 1-\epsilon_{n} \bar{\gamma}$. Next, we will assume that $\|I-A\| \leq\|1-\bar{\gamma}\|$.

Next, we will divide the proof into six steps.

Step 1. First, we will show that $\left\{x_{n}\right\}$ and $\left\{u_{n}\right\}$ are bounded. Since $B$ is $\beta$-inverse-strongly monotone mappings, we have

$$
\begin{aligned}
\|(I-\lambda B) x-(I-\lambda B) y\|^{2} & =\|I x-\lambda B x-I y+\lambda B y\|^{2} \\
& =\|x-y-\lambda B x+\lambda B y\|^{2} \\
& =\|(x-y)-\lambda(B x+B y)\|^{2} \\
& \leq\|x-y\|^{2}-2 \lambda\langle x-y\rangle\langle B x+B y\rangle+\lambda^{2}\|B x-B y\|^{2} \\
& \leq\|x-y\|^{2}-2 \lambda \beta\|B x+B y\|^{2}+\lambda^{2}\|B x-B y\|^{2} \\
& \leq\|x-y\|^{2}+\lambda(\lambda-2 \beta)\|B x+B y\|^{2},
\end{aligned}
$$

if $0<\lambda<2 \beta$, then $I-\lambda B$ is nonexpansive. 
Put $y_{n}:=J_{M, \lambda}\left(u_{n}-\lambda B u_{n}\right), n \geq 0$. Since $J_{M, \lambda}$ and $I-\lambda B$ are nonexpansive mapping, it follows that

$$
\begin{aligned}
\left\|y_{n}-q\right\| & =\left\|J_{M, \lambda}\left(u_{n}-\lambda B u_{n}\right)-J_{M, \lambda}(q-\lambda B q)\right\| \\
& \leq\left\|\left(u_{n}-\lambda B u_{n}\right)-(q-\lambda B q)\right\| \\
& \leq\left\|u_{n}-q\right\| .
\end{aligned}
$$

By Lemma 2.7, we have

$$
\begin{gathered}
u_{n}=K_{r_{n}, n}^{F_{N}} \cdot K_{r_{n-1}, n}^{F_{N-1}} \cdot K_{r_{n-2}, n}^{F_{N-2}} \cdots \cdots K_{r_{2}, n}^{F_{2}} \cdot K_{r_{1}, n}^{F_{1}} \cdot x_{n}, \quad \text { for } n \geq 0 \\
\tau_{n}^{k}=K_{r_{k}, n}^{F_{k}} \cdot K_{r_{k-1}, n}^{F_{k-1}} \cdots \cdots K_{r_{2}, n}^{F_{2}} \cdot K_{r_{1}, n}^{F_{1}} \text { for } k \in\{0,1,2, \ldots, N\},
\end{gathered}
$$

and $\tau_{n}^{0}=I$ for all $n \in N, q=\tau_{r_{k}, n}^{F_{k}} q, u_{n}=\tau_{r_{k}, N}^{N} x_{n}$ Then, we have

$$
\begin{aligned}
\left\|u_{n}-q\right\|^{2} & =\left\|\tau_{r_{k}, n}^{N} x_{n}-\tau_{r_{k}, n}^{F_{k}} q\right\|^{2} \\
& =\left\|x_{n}-q\right\|^{2} .
\end{aligned}
$$

Hence, we get

$$
\left\|y_{n}-q\right\| \leq\left\|x_{n}-q\right\|
$$

From (3.2), we deduce that

$$
\begin{aligned}
\left\|x_{n+1}-q\right\|= & \left\|P_{C}\left(\epsilon_{n} \gamma f\left(x_{n}\right)+\left(I-\epsilon_{n} A\right) W_{n} y_{n}\right)-P_{C} q\right\| \\
\leq & \left\|\epsilon_{n}\left(\gamma f\left(x_{n}\right)-A q\right)+\left(I-\epsilon_{n} A\right)\left(W_{n} y_{n}-q\right)\right\| \\
\leq & \epsilon_{n}\left\|\gamma f\left(x_{n}\right)-A q\right\|+\left(1-\epsilon_{n} \bar{\gamma}\right)\left\|y_{n}-q\right\| \\
\leq & \epsilon \gamma \epsilon_{n}\left\|x_{n}-q\right\|+\epsilon_{n}\|\gamma f(g)-A q\| \\
& +\left(1-\epsilon_{n} \bar{\gamma}\right)\left\|x_{n}-q\right\| \\
= & \left(1-(\bar{\gamma}-\gamma \epsilon) \epsilon_{n}\right)\left\|x_{n}-q\right\|-\epsilon_{n}\|\gamma f(q)-A q\| \\
= & \left(1-(\bar{\gamma}-\gamma \epsilon) \epsilon_{n}\right)\left\|x_{n}-q\right\|+(\bar{\gamma}-\gamma \epsilon) \epsilon_{n} \frac{\|\gamma f(q)-A q\|}{\bar{\gamma}-\gamma \epsilon} \\
& \vdots \\
\leq & \max \left\{\left\|x_{n}-q\right\|, \frac{\|\gamma f(q)-A q\|}{\bar{\gamma}-\gamma \epsilon}\right\} .
\end{aligned}
$$


Journal of Applied Mathematics

It follows by induction that

$$
\left\|x_{n}-q\right\| \leq \max \left\{\left\|x_{0}-q\right\|, \frac{\|\gamma f(q)-A q\|}{\bar{\gamma}-\gamma \epsilon}\right\}, \quad n \geq 0 .
$$

Therefore $\left\{x_{n}\right\}$ is bounded, so are $\left\{y_{n}\right\},\left\{B u_{n}\right\},\left\{f\left(x_{n}\right)\right\}$, and $\left\{A W_{n} y_{n}\right\}$.

Step 2. We claim that $\lim _{n \rightarrow \infty}\left\|x_{n+1}-x_{n}\right\|=0$ and $\lim _{n \rightarrow \infty}\left\|y_{n+1}-y_{n}\right\|=0$. From (3.2), we have

$$
\begin{aligned}
\left\|x_{n+1}-x_{n}\right\|= & \left\|P_{C}\left(\epsilon_{n} \gamma f\left(x_{n}\right)+\left(I-\epsilon_{n} A\right) W_{n} y_{n}\right)-P_{C}\left(\epsilon_{n-1} \gamma f\left(x_{n-1}\right)+\left(I-\epsilon_{n-1} A\right) W_{n} y_{n-1}\right)\right\| \\
\leq & \|\left(I-\epsilon_{n} A\right)\left(W_{n} y_{n}-W_{n} y_{n-1}\right)-\left(\epsilon_{n}-\epsilon_{n-1}\right) A W_{n} y_{n-1} \\
& +\gamma \epsilon_{n}\left(f\left(x_{n}\right)-f\left(x_{n-1}\right)\right)+\gamma\left(\epsilon_{n}-\epsilon_{n-1}\right) f\left(x_{n-1}\right) \| \\
\leq & \left(1-\epsilon_{n} \bar{\gamma}\right)\left\|y_{n}-y_{n-1}\right\|+\left|\epsilon_{n}-\epsilon_{n-1}\right|\left\|A W_{n} y_{n}\right\|+\gamma \epsilon \epsilon_{n}\left\|x_{n}-x_{n-1}\right\| \\
& +\gamma\left|\epsilon_{n}-\epsilon_{n-1}\right|\left\|f\left(x_{n-1}\right)\right\| .
\end{aligned}
$$

Since $J_{M, \lambda}$ and $I-\lambda B$ are nonexpansive, we also have

$$
\begin{aligned}
\left\|y_{n}-y_{n-1}\right\| & =\left\|J_{M, \lambda}\left(u_{n}-\lambda B u_{n}\right)-J_{M, \lambda}\left(u_{n-1}-\lambda B u_{n-1}\right)\right\| \\
& \leq\left\|\left(u_{n}-\lambda B u_{n}\right)-\left(u_{n-1}-\lambda B u_{n-1}\right)\right\| \\
& \leq\left\|u_{n}-u_{n-1}\right\| .
\end{aligned}
$$

On the other hand, from $u_{n-1}=\tau_{r_{k}, n-1}^{N} x_{n-1}$ and $u_{n}=\tau_{r_{k}, n}^{N} x_{n}$, it follows that

$$
\begin{gathered}
F\left(u_{n-1}, y\right)+\varphi(y)-\varphi\left(u_{n-1}\right)+\frac{1}{r_{n-1}}\left\langle y-u_{n-1}, u_{n-1}-x_{n-1}\right\rangle \geq 0, \quad \forall y \in C, \\
F\left(u_{n}, y\right)+\varphi(y)-\varphi\left(u_{n}\right)+\frac{1}{r_{n}}\left\langle y-u_{n}, u_{n}-x_{n}\right\rangle \geq 0, \quad \forall y \in C
\end{gathered}
$$

Substituting $y=u_{n}$ into (3.15) and $y=u_{n-1}$ into (3.16), we get

$$
\begin{gathered}
F\left(u_{n-1}, u_{n}\right)+\varphi\left(u_{n}\right)-\varphi\left(u_{n-1}\right)+\frac{1}{r_{n-1}}\left\langle u_{n}-u_{n-1}, u_{n-1}-x_{n-1}\right\rangle \geq 0, \\
F\left(u_{n}, u_{n+1}\right)+\varphi\left(u_{n+1}\right)-\varphi\left(u_{n}\right)+\frac{1}{r_{n}}\left\langle u_{n+1}-u_{n}, u n-x_{n}\right\rangle \geq 0 .
\end{gathered}
$$


From (A2), we obtain

$$
\begin{gathered}
\left\langle u_{n}-u_{n-1}, \frac{u_{n-1}-x_{n-1}}{r_{n-1}}-\frac{u_{n}-x_{n}}{r_{n}}\right\rangle \geq 0, \\
\left\langle u_{n}-u_{n-1}, u_{n-1}-x_{n-1}-\frac{r_{n-1}}{r_{n}}\left(u_{n}-x_{n}\right)\right\rangle \geq 0,
\end{gathered}
$$

so,

$$
\left\langle u_{n}-u_{n-1}, u_{n-1}-u_{n}+u_{n}-x_{n-1}-\frac{r_{n-1}}{r_{n}}\left(u_{n}-x_{n}\right)\right\rangle \geq 0
$$

It follows that

$$
\begin{gathered}
\left\langle u_{n}-u_{n-1}, u_{n-1}-u_{n}+u_{n}-x_{n}-\frac{r_{n-1}}{r_{n}}\left(u_{n}-x_{n}\right)\right\rangle \geq 0, \\
\left\langle u_{n}-u_{n-1}, u_{n-1}-u_{n}\right\rangle+\left\langle u_{n}-u_{n-1},\left(1-\frac{r_{n-1}}{r_{n}}\right)\left(u_{n}-x_{n}\right)\right\rangle \geq 0 .
\end{gathered}
$$

Without loss of generality, let us assume that there exists a real number $c$ such that $r_{n-1}>c>$ 0 , for all $n \in \mathbb{N}$. Then, we have

$$
\begin{aligned}
\left\|u_{n}-u_{n-1}\right\|^{2} & \leq\left\langle u_{n}-u_{n-1},\left(1-\frac{r_{n-1}}{r_{n}}\right)\left(u_{n}-x_{n}\right)\right\rangle \\
& \leq\left\|u_{n}-u_{n-1}\right\|\left\{\left|1-\frac{r_{n-1}}{r_{n}}\right|\left\|u_{n}-x_{n}\right\|\right\},
\end{aligned}
$$

and hence

$$
\begin{aligned}
\left\|u_{n}-u_{n-1}\right\| & \leq\left\|x_{n}-x_{n-1}\right\|+\frac{1}{r_{n}}\left|r_{n}-r_{n-1}\right|\left\|u_{n}-x_{n}\right\| \\
& \leq\left\|x_{n}-x_{n-1}\right\|+\frac{M_{1}}{c}\left|r_{n}-r_{n-1}\right|
\end{aligned}
$$

where $M_{1}=\sup \left\{\left\|u_{n}-x_{n}\right\|: n \in \mathbb{N}\right\}$. Substituting (3.22) into (3.14), we have

$$
\left\|y_{n}-y_{n-1}\right\| \leq\left\|x_{n}-x_{n-1}\right\|+\frac{M_{1}}{c}\left|r_{n}-r_{n-1}\right|
$$


Substituting (3.23) into (3.13), we get

$$
\begin{aligned}
\left\|x_{n+1}-x_{n}\right\| \leq & \left(1-\epsilon_{n} \bar{\gamma}\right)\left(\left\|x_{n}-x_{n-1}\right\|+\frac{M_{1}}{c}\left|r_{n}-r_{n-1}\right|\right)+\left|\epsilon_{n}-\epsilon_{n-1}\right|\left\|A W_{n} y_{n-1}\right\| \\
& +\gamma \epsilon \epsilon_{n}\left\|x_{n}-x_{n-1}\right\|+\gamma\left|\epsilon_{n}-\epsilon_{n-1}\right|\left\|f\left(x_{n-1}\right)\right\| \\
= & \left(1-\epsilon_{n} \bar{\gamma}\right)\left\|x_{n}-x_{n-1}\right\|+\left(1-\epsilon_{n} \bar{\gamma}\right) \frac{M_{1}}{c}\left|r_{n}-r_{n-1}\right|+\left|\epsilon_{n}-\epsilon_{n-1}\right|\left\|A W_{n} y_{n-1}\right\| \\
& +\gamma \epsilon \epsilon_{n}\left\|x_{n}-x_{n-1}\right\|+\gamma\left|\epsilon_{n}-\epsilon_{n-1}\right|\left\|f\left(x_{n-1}\right)\right\| \\
\leq & \left(1-(\bar{\gamma}-\gamma \epsilon) \epsilon_{n}\right)\left\|x_{n}-x_{n-1}\right\|+\frac{M_{1}}{c}\left|r_{n}-r_{n-1}\right|+\left|\epsilon_{n}-\epsilon_{n-1}\right|\left\|A W_{n} y_{n-1}\right\| \\
& +\gamma\left|\epsilon_{n}-\epsilon_{n-1}\right|\left\|f\left(x_{n-1}\right)\right\| \\
\leq & \left(1-(\bar{\gamma}-\gamma \epsilon) \epsilon_{n}\right)\left\|x_{n}-x_{n-1}\right\|+\frac{M_{1}}{c}\left|r_{n}-r_{n-1}\right|+M_{2}\left|\epsilon_{n}-\epsilon_{n-1}\right|,
\end{aligned}
$$

where $M_{2}=\sup \left\{\max \left\{\left\|A W_{n} y_{n-1}\right\|,\left\|f\left(x_{n-1}\right)\right\|: n \in \mathbb{N}\right\}\right\}$. Since conditions (C1)-(C2) and by Lemma 2.5, we have $\left\|x_{n+1}-x_{n}\right\| \rightarrow 0$ as $n \rightarrow \infty$. From (3.23), we also have $\left\|y_{n+1}-y_{n}\right\| \rightarrow 0$ as $n \rightarrow \infty$.

Step 3. Next, we show that $\lim _{n \rightarrow \infty}\left\|B u_{n}-B q\right\|=0$.

For $q \in \theta$ hence $q=J_{M, \lambda}(q-\lambda B q)$. By (3.6) and (3.9), we get

$$
\begin{aligned}
\left\|y_{n}-q\right\|^{2} & =\left\|J_{M, \lambda}\left(u_{n}-\lambda B u_{n}\right)-J_{M, \lambda}(q-\lambda B q)\right\|^{2} \\
& \leq\left\|\left(u_{n}-\lambda B u_{n}\right)-(q-\lambda B q)\right\|^{2} \\
& \leq\left\|u_{n}-q\right\|^{2}+\lambda(\lambda-2 \beta)\left\|B u_{n}-B q\right\|^{2} \\
& \leq\left\|x_{n}-q\right\|^{2}+\lambda(\lambda-2 \beta)\left\|B u_{n}-B q\right\|^{2} .
\end{aligned}
$$

It follows that

$$
\begin{aligned}
\left\|x_{n+1}-q\right\|^{2}= & \left\|P_{C}\left(\epsilon_{n} \gamma f\left(x_{n}\right)+\left(I-\epsilon_{n} A\right) W_{n} y_{n}\right)-P_{C}(q)\right\|^{2} \\
\leq & \left\|\epsilon_{n}\left(\gamma f\left(x_{n}\right)-A q\right)+\left(I-\epsilon_{n} A\right)\left(W_{n} y_{n}-q\right)\right\|^{2} \\
\leq & \left(\epsilon_{n}\left\|\gamma f\left(x_{n}\right)-A q\right\|+\left(1-\epsilon_{n} \bar{\gamma}\right)\left\|y_{n}-q\right\|\right)^{2} \\
\leq & \epsilon_{n}\left\|\gamma f\left(x_{n}\right)-A q\right\|^{2}+\left(1-\epsilon_{n} \bar{\gamma}\right)\left\|y_{n}-q\right\|^{2} \\
& +2 \epsilon_{n}\left(1-\epsilon_{n} \bar{\gamma}\right)\left\|\gamma f\left(x_{n}\right)-A q\right\|\left\|y_{n}-q\right\|
\end{aligned}
$$




$$
\begin{aligned}
\leq & \epsilon_{n}\left\|\gamma f\left(x_{n}\right)-A q\right\|^{2}+2 \epsilon_{n}\left(1-\epsilon_{n} \bar{\gamma}\right)\left\|\gamma f\left(x_{n}\right)-A q\right\|\left\|y_{n}-q\right\| \\
& +\left(1-\epsilon_{n} \bar{\gamma}\right)\left(\left\|x_{n}-q\right\|^{2}+\lambda(\lambda-2 \beta)\left\|B u_{n}-B q\right\|^{2}\right) \\
\leq & \epsilon_{n}\left\|\gamma f\left(x_{n}\right)-A q\right\|^{2}+2 \epsilon_{n}\left(1-\epsilon_{n} \bar{\gamma}\right)\left\|\gamma f\left(x_{n}\right)-A q\right\|\left\|y_{n}-q\right\| \\
& +\left\|x_{n}-q\right\|^{2}+\left(1-\epsilon_{n} \bar{\gamma}\right) \lambda(\lambda-2 \beta)\left\|B u_{n}-B q\right\|^{2} .
\end{aligned}
$$

So, we obtain

$$
\begin{aligned}
\left(1-\epsilon_{n} \bar{\gamma}\right) \lambda(2 \beta-\lambda)\left\|B u_{n}-B q\right\|^{2} \leq & \epsilon_{n}\left\|\gamma f\left(x_{n}\right)-A q\right\|^{2} \\
& +\left\|x_{n}-x_{n+1}\right\|\left(\left\|x_{n}-q\right\|+\left\|x_{n+1}-q\right\|\right)+\xi_{n},
\end{aligned}
$$

where $\xi_{n}=2 \epsilon_{n}\left(1-\epsilon_{n} \bar{\gamma}\right)\left\|\gamma f\left(x_{n}\right)-A q\right\|\left\|y_{n}-q\right\|$. By conditions (C1), (C3) and $\lim _{n \rightarrow \infty}\left\|x_{n+1}-x_{n}\right\|=$ 0 , then, we obtain that $\left\|B u_{n}-B q\right\| \rightarrow 0$ as $n \rightarrow \infty$.

Step 4. We show the following:

(i) $\lim _{n \rightarrow \infty}\left\|x_{n}-u_{n}\right\|=0$;

(ii) $\lim _{n \rightarrow \infty}\left\|u_{n}-y_{n}\right\|=0$;

(iii) $\lim _{n \rightarrow \infty}\left\|y_{n}-W_{n} y_{n}\right\|=0$.

Since $K_{r_{n}}(x)$ is firmly nonexpansive and (2.3), we observe that

$$
\begin{aligned}
\left\|u_{n}-q\right\|^{2} & =\left\|\tau_{r_{n}, n}^{N} x_{n}-\tau_{r_{n}, n}^{N} q\right\|^{2} \\
& \leq\left\langle x_{n}-q, u_{n}-q\right\rangle \\
& =\frac{1}{2}\left(\left\|x_{n}-q\right\|^{2}+\left\|u_{n}-q\right\|^{2}-\left\|x_{n}-q-u_{n}-q\right\|^{2}\right) \\
& \leq \frac{1}{2}\left(\left\|x_{n}-q\right\|^{2}+\left\|u_{n}-q\right\|^{2}-\left\|x_{n}-u_{n}\right\|^{2}\right),
\end{aligned}
$$

it follows that

$$
\left\|u_{n}-q\right\|^{2} \leq\left\|x_{n}-q\right\|^{2}-\left\|x_{n}-u_{n}\right\|^{2} .
$$


Journal of Applied Mathematics

Since $J_{M, \lambda}$ is 1-inverse-strongly monotone and by (2.3), we compute

$$
\begin{aligned}
\left\|y_{n}-q\right\|^{2}= & \left\|J_{M, \lambda}\left(u_{n}-\lambda B u_{n}\right)-J_{M, \lambda}(q-\lambda B q)\right\|^{2} \\
\leq & \left\langle\left(u_{n}-\lambda B u_{n}\right)-(q-\lambda B q), y_{n}-q\right\rangle \\
= & \frac{1}{2}\left(\left\|\left(u_{n}-\lambda B u_{n}\right)-(q-\lambda B q)\right\|^{2}+\left\|y_{n}-q\right\|^{2}\right. \\
& \left.-\left\|\left(u_{n}-\lambda B u_{n}\right)-(q-\lambda B q)-\left(y_{n}-q\right)\right\|^{2}\right) \\
\leq & \frac{1}{2}\left(\left\|u_{n}-q\right\|^{2}+\left\|y_{n}-q\right\|^{2}-\left\|\left(u_{n}-y_{n}\right)-\lambda\left(B u_{n}-B q\right)\right\|^{2}\right) \\
= & \frac{1}{2}\left(\left\|u_{n}-q\right\|^{2}+\left\|y_{n}-q\right\|^{2}-\left\|u_{n}-y_{n}\right\|^{2}\right. \\
& \left.+2 \lambda\left\langle u_{n}-y_{n}, B u_{n}-B q\right\rangle-\lambda^{2}\left\|B u_{n}-B q\right\|^{2}\right),
\end{aligned}
$$

which implies that

$$
\left\|y_{n}-q\right\|^{2} \leq\left\|u_{n}-q\right\|^{2}-\left\|u_{n}-y_{n}\right\|^{2}+2 \lambda\left\|u_{n}-y_{n}\right\|\left\|B u_{n}-B q\right\| .
$$

Substituting (3.31) into (3.26), we have

$$
\begin{aligned}
\left\|x_{n+1}-q\right\|^{2} \leq & \epsilon_{n}\left\|\gamma f\left(x_{n}\right)-A q\right\|^{2}+\left\|y_{n}-q\right\|^{2}+2 \epsilon_{n}\left(1-\epsilon_{n} \bar{\gamma}\right)\left\|\gamma f\left(x_{n}\right)-A q\right\|\left\|y_{n}-q\right\| \\
\leq & \epsilon_{n}\left\|\gamma f\left(x_{n}\right)-A q\right\|^{2}+\left(\left\|u_{n}-q\right\|^{2}-\left\|u_{n}-y_{n}\right\|^{2}+2 \lambda_{n}\left\|u_{n}-y_{n}\right\|\left\|B u_{n}-B q\right\|\right) \\
& +2 \epsilon_{n}\left(1-\epsilon_{n} \bar{\gamma}\right)\left\|\gamma f\left(x_{n}\right)-A q\right\|\left\|y_{n}-q\right\| .
\end{aligned}
$$

Then, we derive

$$
\begin{aligned}
\left\|x_{n}-u_{n}\right\|^{2}+\left\|u_{n}-y_{n}\right\|^{2} \leq & \epsilon_{n}\left\|\gamma f\left(x_{n}\right)-A q\right\|^{2}+\left\|x_{n}-q\right\|^{2}-\left\|x_{n+1}-q\right\|^{2} \\
& +2 \lambda\left\|u_{n}-y_{n}\right\|\left\|B u_{n}-B q\right\|+2 \epsilon_{n}\left(1-\epsilon_{n} \bar{\gamma}\right)\left\|\gamma f\left(x_{n}\right)-A q\right\|\left\|y_{n}-q\right\| . \\
= & \epsilon_{n}\left\|\gamma f\left(x_{n}\right)-A q\right\|^{2}+\left\|x_{n}-x_{n+1}\right\|\left(\left\|x_{n}-q\right\|+\left\|x_{n+1}-q\right\|\right) \\
& +2 \lambda\left\|u_{n}-y_{n}\right\|\left\|B u_{n}-B q\right\|+2 \epsilon_{n}\left(1-\epsilon_{n} \bar{\gamma}\right)\left\|\gamma f\left(x_{n}\right)-A q\right\|\left\|y_{n}-q\right\| .
\end{aligned}
$$

By condition (C1), $\lim _{n \rightarrow \infty}\left\|x_{n}-x_{n+1}\right\|=0$ and $\lim _{n \rightarrow \infty}\left\|B u_{n}-B q\right\|=0$.

So, we have $\left\|x_{n}-u_{n}\right\| \rightarrow 0,\left\|u_{n}-y_{n}\right\| \rightarrow 0$ as $n \rightarrow \infty$. It follows that

$$
\left\|x_{n}-y_{n}\right\| \leq\left\|x_{n}-u_{n}\right\|+\left\|u_{n}-y_{n}\right\| \longrightarrow 0, \quad \text { as } n \longrightarrow \infty
$$


From (3.2), we have

$$
\begin{aligned}
\left\|x_{n}-W_{n} y_{n}\right\| & \leq\left\|x_{n}-W_{n} y_{n-1}\right\|+\left\|W_{n} y_{n-1}-W_{n} y_{n}\right\| \\
& \leq\left\|P_{C}\left(\epsilon_{n-1} \gamma f\left(x_{n-1}\right)+\left(I-\alpha_{n-1} A\right) W_{n} y_{n-1}\right)-P_{C}\left(W_{n} y_{n-1}\right)\right\|+\left\|y_{n-1}-y_{n}\right\| \\
& \leq \epsilon_{n-1}\left\|\gamma f x_{n-1}-A W_{n} y_{n-1}\right\|+\left\|y_{n-1}-y_{n}\right\| .
\end{aligned}
$$

By condition $(\mathrm{C} 1)$ and $\lim _{n \rightarrow \infty}\left\|y_{n-1}-y_{n}\right\|=0$, we obtain that $\left\|x_{n}-W_{n} y_{n}\right\| \rightarrow 0$ as $n \rightarrow \infty$.

Hence, we have

$$
\begin{aligned}
\left\|x_{n}-W_{n} x_{n}\right\| & \leq\left\|x_{n}-W_{n} y_{n}\right\|+\left\|W_{n} y_{n}-W_{n} x_{n}\right\| \\
& \leq\left\|x_{n}-W_{n} y_{n}\right\|+\left\|y_{n}-x_{n}\right\| .
\end{aligned}
$$

By (3.34) and $\lim _{n \rightarrow \infty}\left\|x_{n}-W_{n} y_{n}\right\|=0$, we obtain $\left\|x_{n}-W_{n} x_{n}\right\| \rightarrow 0$ as $n \rightarrow \infty$.

Moreover, we also have

$$
\left\|y_{n}-W_{n} y_{n}\right\| \leq\left\|y_{n}-x_{n}\right\|+\left\|x_{n}-W_{n} y_{n}\right\|
$$

By (3.34) and $\lim _{n \rightarrow \infty}\left\|x_{n}-W_{n} y_{n}\right\|=0$, we obtain $\left\|y_{n}-W_{n} y_{n}\right\| \rightarrow 0$ as $n \rightarrow \infty$.

Step 5. We show that $q \in \theta:=\bigcap_{n=1}^{\infty} F\left(T_{n}\right) \cap\left(\bigcap_{k=1}^{N} \operatorname{SMEP}\left(F_{k}\right)\right) \cap I(B, M)$ and $\limsup _{n \rightarrow \infty}\langle(\gamma f-$ A) $\left.q, W_{n} y_{n}-q\right\rangle \leq 0$. It is easy to see that $P_{\theta}(\gamma f+(I-A))$ is a contraction of $H$ into itself. Indeed, since $0<\gamma<\bar{\gamma} / \epsilon$, we have

$$
\begin{aligned}
\left\|P_{\theta}(\gamma f+(I-A)) x-P_{\theta}(\gamma f+(I-A)) y\right\| & \leq \gamma\|f(x)-f(y)\|+\|I-A\|\|x-y\| \\
& \leq \gamma \epsilon\|x-y\|+(1-\bar{\gamma})\|x-y\| \\
& \leq(1-\bar{\gamma}+\gamma \epsilon)\|x-y\| .
\end{aligned}
$$

Since $H$ is complete, then there exists a unique fixed point $q \in H$ such that $q=P_{\theta}(\gamma f+(I-$ $A))(q)$. By Lemma 2.2, we obtain that $\langle(\gamma f-A) q, w-q\rangle \leq 0$ for all $w \in \theta$.

Next, we show that $\limsup _{n \rightarrow \infty}\left\langle(\gamma f-A) q, W_{n} y_{n}-q\right\rangle \leq 0$, where $q=P_{\theta}(\gamma f+I-A)(q)$ is the unique solution of the variational inequality $\langle(\gamma f-A) q, w-q\rangle \geq 0$ for all $w \in \theta$. We can choose a subsequence $\left\{y_{n_{i}}\right\}$ of $\left\{y_{n}\right\}$ such that

$$
\limsup _{n \rightarrow \infty}\left\langle(\gamma f-A) q, W_{n} y_{n}-q\right\rangle=\lim _{i \rightarrow \infty}\left\langle(\gamma f-A) q, W_{n} y_{n_{i}}-q\right\rangle
$$

As $\left\{y_{n_{i}}\right\}$ is bounded, there exists a subsequence $\left\{y_{n_{i j}}\right\}$ of $\left\{y_{n_{i}}\right\}$ which converges weakly to $w$. We may assume without loss of generality that $y_{n_{i}} \rightarrow w$.

Next we claim that $w \in \theta$. Since $\left\|y_{n}-W_{n} y_{n}\right\| \rightarrow 0,\left\|x_{n}-W_{n} x_{n}\right\| \rightarrow 0$, and $\left\|x_{n}-y_{n}\right\| \rightarrow$ 0 , and by Lemma 2.6, we have $w \in \bigcap_{n=1}^{\infty} F\left(T_{n}\right)$. 

know that

Next, we show that $w \in \bigcap_{k=1}^{\infty} \operatorname{SMEP}\left(F_{k}\right)$. Since $u_{n}=\tau_{r_{k}, n}^{N} x_{n}$, for $k=1,2,3, \ldots, N$, we

$$
F_{k}\left(u_{n}, y\right)+\varphi(y)-\varphi\left(u_{n}\right)+\frac{1}{r_{n}}\left\langle y-u_{n}, u_{n}-x_{n}\right\rangle \geq 0, \quad \forall y \in C
$$

It follows by (A2) that

$$
\varphi(y)-\varphi\left(u_{n}\right)+\frac{1}{r_{n}}\left\langle y-u_{n}, u_{n}-x_{n}\right\rangle \geq F_{k}\left(y, u_{n}\right), \quad \forall y \in C
$$

Hence, for $k=1,2,3, \ldots, N$, we get

$$
\varphi(y)-\varphi\left(u_{n_{i}}\right)+\frac{1}{r_{n_{i}}}\left\langle y-u_{n_{i}}, u_{n_{i}}-x_{n_{i}}\right\rangle \geq F_{k}\left(y, u_{n_{i}}\right), \quad \forall y \in C
$$

For $t \in(0,1]$ and $y \in H$, let $y_{t}=t y+(1-t) w$. From (3.42), we have

$$
0 \geq \varphi\left(y_{t}\right)+\varphi\left(u_{n_{i}}\right)-\frac{1}{r_{n_{i}}}\left\langle y_{t}-u_{n_{i}}, u_{n_{i}}-x_{n_{i}}\right\rangle+F_{k}\left(y_{t}, u_{n_{i}}\right) .
$$

Since $\left\|u_{n_{i}}-x_{n_{i}}\right\| \rightarrow 0$, from (A4) and the weakly lower semicontinuity of $\varphi,\left(u_{n_{i}}-\right.$ $\left.x_{n_{i}}\right) / r_{n_{i}} \rightarrow 0$ and $u_{n_{i}} \rightarrow w$. From (A1) and (A4), we have

$$
\begin{aligned}
0 & =F_{k}\left(y_{t}, y_{t}\right)-\varphi\left(y_{t}\right)+\varphi\left(y_{t}\right) \\
& \leq t F_{k}\left(y_{t}, y\right)+(1-t) F_{k}\left(y_{t}, w\right)+t \varphi(y)+(1-t) \varphi(w)-\varphi\left(y_{t}\right) \\
& \leq t\left[F_{k}\left(y_{t}, y\right)+\varphi(y)-\varphi\left(y_{t}\right)\right] .
\end{aligned}
$$

Dividing by $t$, we get

$$
F_{k}\left(y_{t}, y\right)+\varphi(y)-\varphi\left(y_{t}\right) \geq 0
$$

The weakly lower semicontinuity of $\varphi$ for $k=1,2,3, \ldots, N$, we get

$$
F_{k}(w, y)+\varphi(y) \geq \varphi(w)
$$

So, we have

$$
F_{k}(w, y)+\varphi(y)-\varphi(w) \geq 0, \quad \forall k=1,2,3, \ldots, N
$$

This implies that $w \in \bigcap_{k=1}^{N} \operatorname{SMEP}\left(F_{k}\right)$.

Lastly, we show that $w \in I(B, M)$. In fact, since B is $\beta$-inverse strongly monotone, hence $B$ is a monotone and Lipschitz continuous mapping. It follows from Lemma 2.3 that 
$M+B$ is a maximal monotone. Let $(v, g) \in G(M+B)$, since $g-B v \in M(v)$. Again since $y_{n_{i}}=J_{M, \lambda}\left(u_{n_{i}}-\lambda B u_{n_{i}}\right)$, we have $u_{n_{i}}-\lambda B u_{n_{i}} \in(I+\lambda M)\left(y_{n_{i}}\right)$, that is, $(1 / \lambda)\left(u_{n_{i}}-y_{n_{i}}-\lambda B u_{n_{i}}\right) \in$ $M\left(y_{n_{i}}\right)$. By virtue of the maximal monotonicity of $M+B$, we have

$$
\left\langle v-y_{n_{i}}, g-B v-\frac{1}{\lambda}\left(u_{n_{i}}-y_{n_{i}}-\lambda B u_{n_{i}}\right)\right\rangle \geq 0
$$

and hence

$$
\begin{aligned}
\left\langle v-y_{n_{i}}, g\right\rangle & \geq\left\langle v-y_{n_{i}}, B v+\frac{1}{\lambda}\left(u_{n_{i}}-y_{n_{i}}-\lambda B u_{n_{i}}\right)\right\rangle \\
& =\left\langle v-y_{n_{i}}, B v-B y_{n_{i}}\right\rangle+\left\langle v-y_{n_{i}}, B y_{n_{i}}-B u_{n_{i}}\right\rangle+\left\langle v-y_{n_{i}}, \frac{1}{\lambda}\left(u_{n_{i}}-y_{n_{i}}\right)\right\rangle .
\end{aligned}
$$

It follows from $\lim _{n \rightarrow \infty}\left\|u_{n}-y_{n}\right\|=0$, we have $\lim _{n \rightarrow \infty}\left\|B u_{n}-B y_{n}\right\|=0$ and $y_{n_{i}} \rightarrow w$, it follows that

$$
\limsup _{n \rightarrow \infty}\left\langle v-y_{n}, g\right\rangle=\langle v-w, g\rangle \geq 0
$$

It follows from the maximal monotonicity of $B+M$ that $\theta \in(M+B)(w)$, that is, $w \in I(B, M)$. Therefore, $w \in \theta$. We observe that

$$
\limsup _{n \rightarrow \infty}\left\langle(\gamma f-A) q, W_{n} y_{n}-q\right\rangle=\lim _{i \rightarrow \infty}\left\langle(\gamma f-A) q, W_{n} y_{n_{i}}-q\right\rangle=\langle(\gamma f-A) q, w-q\rangle \leq 0 .
$$

Step 6. Finally, we prove $x_{n} \rightarrow q$. By using (3.2) and together with Schwarz inequality, we have

$$
\begin{aligned}
\left\|x_{n+1}-q\right\|^{2}= & \left\|P_{C}\left(\epsilon_{n} \gamma f\left(x_{n}\right)+\left(I-\epsilon_{n} A\right) W_{n} y_{n}\right)-P_{C}(q)\right\|^{2} \\
\leq & \left\|\epsilon_{n}\left(\gamma f\left(x_{n}\right)-A q\right)+\left(I-\epsilon_{n} A\right)\left(W_{n} y_{n}-q\right)\right\|^{2} \\
\leq & \left(I-\epsilon_{n} A\right)^{2}\left\|\left(W_{n} y_{n}-q\right)\right\|^{2}+\epsilon_{n}^{2}\left\|\gamma f\left(x_{n}\right)-A q\right\|^{2} \\
& +2 \epsilon_{n}\left\langle\left(I-\epsilon_{n} A\right)\left(W_{n} y_{n}-q\right), \gamma f\left(x_{n}\right)-A q\right\rangle \\
\leq & \left(1-\epsilon_{n} \bar{\gamma}\right)^{2}\left\|y_{n}-q\right\|^{2}+\epsilon_{n}^{2}\left\|\gamma f\left(x_{n}\right)-A q\right\|^{2} \\
& +2 \epsilon_{n}\left\langle W_{n} y_{n}-q, \gamma f\left(x_{n}\right)-A q\right\rangle-2 \epsilon_{n}^{2}\left\langle A\left(W_{n} y_{n}-q\right), \gamma f\left(x_{n}\right)-A q\right\rangle \\
\leq & \left(1-\epsilon_{n} \bar{\gamma}\right)^{2}\left\|x_{n}-q\right\|^{2}+\epsilon_{n}^{2}\left\|\gamma f\left(x_{n}\right)-A q\right\|^{2}+2 \epsilon_{n}\left\langle W_{n} y_{n}-q, \gamma f\left(x_{n}\right)-\gamma f(q)\right\rangle \\
& +2 \epsilon_{n}\left\langle W_{n} y_{n}-q, \gamma f(q)-A q\right\rangle-2 \epsilon_{n}^{2}\left\langle A\left(W_{n} y_{n}-q\right), \gamma f\left(x_{n}\right)-A q\right\rangle
\end{aligned}
$$


Journal of Applied Mathematics

$$
\begin{aligned}
\leq & \left(1-\epsilon_{n} \bar{\gamma}\right)^{2}\left\|x_{n}-q\right\|^{2}+\epsilon_{n}^{2}\left\|\gamma f\left(x_{n}\right)-A q\right\|^{2}+2 \epsilon_{n}\left\|W_{n} y_{n}-q\right\|\left\|\gamma f\left(x_{n}\right)-\gamma f(q)\right\| \\
& +2 \epsilon_{n}\left\langle W_{n} y_{n}-q, \gamma f(q)-A q\right\rangle-2 \epsilon_{n}^{2}\left\langle A\left(W_{n} y_{n}-q\right), \gamma f\left(x_{n}\right)-A q\right\rangle \\
\leq & \left(1-\epsilon_{n} \bar{\gamma}\right)^{2}\left\|x_{n}-q\right\|^{2}+\epsilon_{n}^{2}\left\|\gamma f\left(x_{n}\right)-A q\right\|^{2}+2 \gamma \epsilon \epsilon_{n}\left\|y_{n}-q\right\|\left\|x_{n}-q\right\| \\
& +2 \epsilon_{n}\left\langle W_{n} y_{n}-q, \gamma f(q)-A q\right\rangle-2 \epsilon_{n}^{2}\left\langle A\left(W_{n} y_{n}-q\right), \gamma f\left(x_{n}\right)-A q\right\rangle \\
\leq & \left(1-\epsilon_{n} \bar{\gamma}\right)^{2}\left\|x_{n}-q\right\|^{2}+\epsilon_{n}^{2}\left\|\gamma f\left(x_{n}\right)-A q\right\|^{2}+2 \gamma \epsilon \epsilon_{n}\left\|x_{n}-q\right\|^{2} \\
& +2 \epsilon_{n}\left\langle W_{n} y_{n}-q, \gamma f(q)-A q\right\rangle-2 \epsilon_{n}^{2}\left\langle A\left(W_{n} y_{n}-q\right), \gamma f\left(x_{n}\right)-A q\right\rangle \\
\leq & \left(\left(1-\epsilon_{n} \bar{\gamma}\right)^{2}+2 \gamma \epsilon \epsilon_{n}\right)\left\|x_{n}-q\right\|^{2} \\
& +\epsilon_{n}\left\{\epsilon_{n}\left\|\gamma f\left(x_{n}\right)-A q\right\|^{2}+2\left\langle W_{n} y_{n}-q, \gamma f(q)-A q\right\rangle\right. \\
& \left.\quad-2 \epsilon_{n}\left\|A\left(W_{n} y_{n}-q\right)\right\|\left\|\gamma f\left(x_{n}\right)-A q\right\|\right\} \\
= & \left(1-2(\bar{\gamma}-\gamma \epsilon) \epsilon_{n}\right)\left\|x_{n}-q\right\|^{2} \\
& +\epsilon_{n}\left\{\epsilon_{n}\left\|\gamma f\left(x_{n}\right)-A q\right\|^{2}+2\left\langle W_{n} y_{n}-q, \gamma f(q)-A q\right\rangle\right. \\
& \quad-2 \epsilon_{n}\left\|A\left(W_{n} y_{n}-q\right)\right\|\left\|\gamma f\left(x_{n}\right)-A q\right\| \\
& \left.+\epsilon_{n} \bar{\gamma}^{2}\left\|x_{n}-q\right\|^{2}\right\} .
\end{aligned}
$$

Since $\left\{x_{n}\right\}$ is bounded, where $\eta \geq\left\|\gamma f\left(x_{n}\right)-A q\right\|^{2}-2\left\|A\left(W_{n} y_{n}-q\right)\right\|\left\|\gamma f\left(x_{n}\right)-A q\right\|+$ $\bar{\gamma}^{2}\left\|x_{n}-q\right\|^{2}$ for all $n \geq 0$. It follows that

$$
\left\|x_{n+1}-q\right\|^{2} \leq\left(1-2(\bar{\gamma}-\gamma \epsilon) \epsilon_{n}\right)\left\|x_{n}-q\right\|^{2}+\epsilon_{n} \delta_{n}
$$

where $\delta_{n}=2\left\langle W_{n} y_{n}-q, \gamma f(q)-A q\right\rangle+\eta \alpha_{n}$. Since $\lim \sup _{n \rightarrow \infty}\left\langle(\gamma f-A) q, W_{n} y_{n}-q\right\rangle \leq 0$, we get limsup $\operatorname{sum}_{n \rightarrow \infty} \delta_{n} \leq 0$. Applying Lemma 2.5, we can conclude that $x_{n} \rightarrow q$. This completes the proof.

Corollary 3.2. Let $H$ be a real Hilbert space and $C$ a nonempty closed and convex subset of $H$. Let $B$ be $\beta$-inverse-strongly monotone and $\varphi: C \rightarrow R$ a convex and lower semicontinuous function. Let $f: C \rightarrow C$ be a contraction with coefficient $\alpha(0<\alpha<1), M: H \rightarrow 2^{H}$ a maximal monotone mapping, and $\left\{T_{n}\right\}$ a family of nonexpansive mappings of $H$ into itself such that

$$
\theta:=\bigcap_{n=1}^{\infty} F\left(T_{n}\right) \cap\left(\bigcap_{k=1}^{N} \operatorname{SMEP}\left(F_{k}\right)\right) \cap I(B, M) \neq 0 .
$$


Suppose that $\left\{x_{n}\right\}$ is a sequence generated by the following algorithm for $x_{0}, u_{n} \in C$ arbitrarily:

$$
\begin{gathered}
u_{n}=K_{r_{n}, n}^{F_{N}} \cdot K_{r_{n-1}, n}^{F_{N-1}} \cdot K_{r_{n-2}, n}^{F_{N-2}} \cdots \cdots K_{r_{2}, n}^{F_{2}} \cdot K_{r_{1}, n}^{F_{1}} \cdot x_{n}, \quad \forall n \in N \\
x_{n+1}=P_{C}\left[\epsilon_{n} f\left(x_{n}\right)+\left(I-\epsilon_{n}\right) W_{n} J_{M, \lambda}\left(u_{n}-\lambda B u_{n}\right)\right]
\end{gathered}
$$

for all $n=0,1,2, \ldots$, and the conditions (C1)-(C3) in Theorem 3.1 are satisfied.

Then, the sequence $\left\{x_{n}\right\}$ converges strongly to $q \in \theta$, where $q=P_{\theta}(f+I)(q)$ which solves the following variational inequality:

$$
\langle(f-I) q, p-q\rangle \leq 0, \quad \forall p \in \theta .
$$

Proof. Putting $A \equiv I$ and $\gamma \equiv 1$ in Theorem 3.1, we can obtain the desired conclusion immediately.

Corollary 3.3. Let $H$ be a real Hilbert space and $C$ a nonempty closed and convex subset of $H$. Let $B$ be $\beta$-inverse-strongly monotone, $\varphi: C \rightarrow R$ a convex and lower semicontinuous function, and $M: H \rightarrow 2^{H}$ a maximal monotone mapping. Let $\left\{T_{n}\right\}$ be a family of nonexpansive mappings of $H$ into itself such that

$$
\theta:=\bigcap_{n=1}^{\infty} F\left(T_{n}\right) \cap\left(\bigcap_{k=1}^{N} \operatorname{SMEP}\left(F_{k}\right)\right) \cap I(B, M) \neq 0 .
$$

Suppose that $\left\{x_{n}\right\}$ is a sequence generated by the following algorithm for $x_{0}, u \in C$ and $u_{n} \in C$ :

$$
\begin{gathered}
u_{n}=K_{r_{n}, n}^{F_{N}} \cdot K_{r_{n-1}, n}^{F_{N-1}} \cdot K_{r_{n-2}, n}^{F_{N-2}} \cdots \cdots K_{r_{2}, n}^{F_{2}} \cdot K_{r_{1}, n}^{F_{1}} \cdot x_{n}, \quad \forall n \in N \\
x_{n+1}=P_{C}\left[\epsilon_{n} u+\left(I-\epsilon_{n}\right) W_{n} J_{M, \lambda}\left(u_{n}-\lambda B u_{n}\right)\right]
\end{gathered}
$$

for all $n=0,1,2, \ldots$, and the conditions (C1)-(C3) in Theorem 3.1 are satisfied.

Then, the sequence $\left\{x_{n}\right\}$ converges strongly to $q \in \theta$, where $q=P_{\theta}(q)$ which solves the following variational inequality:

$$
\langle u-q, p-q\rangle \leq 0, \quad \forall p \in \theta .
$$

Proof. Putting $f(x) \equiv u$, for all $x \in C$ in Corollary 3.2, we can obtain the desired conclusion immediately.

Corollary 3.4. Let $H$ be a real Hilbert space and $C$ a nonempty closed and convex subset of $H$, and let $B$ be $\beta$-inverse-strongly monotone mapping and $A$ a strongly positive linear bounded operator of $H$ into itself with coefficient $\bar{\gamma}>0$. Assume that $0<\gamma<\bar{\gamma} / \alpha$. Let $f: C \rightarrow C$ be a contraction with coefficient $\alpha(0<\alpha<1)$ and $\left\{T_{n}\right\}$ be a family of nonexpansive mappings of $H$ into itself such that

$$
\theta:=\bigcap_{n=1}^{\infty} F\left(T_{n}\right) \cap \operatorname{VI}(C, B) \neq \emptyset
$$


Suppose that $\left\{x_{n}\right\}$ is a sequence generated by the following algorithm for $x_{0} \in C$ arbitrarily:

$$
x_{n+1}=P_{C}\left[\epsilon_{n} \gamma f\left(x_{n}\right)+\left(I-\epsilon_{n} A\right) W_{n} P_{C}\left(x_{n}-\lambda B x_{n}\right)\right],
$$

for all $n=0,1,2, \ldots$, and the conditions (C1)-(C3) in Theorem 3.1 are satisfied.

Then, the sequence $\left\{x_{n}\right\}$ converges strongly to $q \in \theta$, where $q=P_{\theta}(\gamma f+I-A)(q)$ which solves the following variational inequality:

$$
\langle(\gamma f-A) q, p-q\rangle \leq 0, \quad \forall p \in \theta
$$

Proof. Taking $F \equiv 0, \varphi \equiv 0, u_{n}=x_{n}$, and $J_{M, \lambda}=P_{C}$ in Theorem 3.1, we can obtain the desired conclusion immediately.

Remark 3.5. Corollary 3.4 generalizes and improves the result of Klin-Eam and Suantai [45].

\section{Applications}

In this section, we apply the iterative scheme (1.25) for finding a common fixed point of nonexpansive mapping and strictly pseudocontractive mapping.

Definition 4.1. A mapping $S: C \rightarrow C$ is called a strictly pseudocontraction if there exists a constant $0 \leq \kappa<1$ such that

$$
\|S x-S y\|^{2} \leq\|x-y\|^{2}+\kappa\|(I-S) x-(I-S) y\|^{2}, \quad \forall x, y \in C
$$

If $\kappa=0$, then $S$ is nonexpansive. In this case, we say that $S: C \rightarrow C$ is a $\kappa$-strictly pseudocontraction. Putting $B=I-S$. Then, we have

$$
\|(I-B) x-(I-B) y\|^{2} \leq\|x-y\|^{2}+\kappa\|B x-B y\|^{2}, \quad \forall x, y \in C .
$$

Observe that

$$
\|(I-B) x-(I-B) y\|^{2}=\|x-y\|^{2}+\|B x-B y\|^{2}-2\langle x-y, B x-B y\rangle, \quad \forall x, y \in C .
$$

Hence, we obtain

$$
\langle x-y, B x-B y\rangle \geq \frac{1-\kappa}{2}\|B x-B y\|^{2}, \quad \forall x, y \in C .
$$

Then, $B$ is a $((1-\kappa) / 2)$-inverse-strongly monotone mapping.

Using Theorem 3.1, we first prove a strongly convergence theorem for finding a common fixed point of a nonexpansive mapping and a strictly pseudocontraction. 
Theorem 4.2. Let $H$ be a real Hilbert space and $C$ a nonempty closed and convex subset of $H$, and let $B$ be an $\beta$-inverse-strongly monotone, $\varphi: C \rightarrow \mathcal{R}$ a convex and lower semicontinuous function, and $f: C \rightarrow C$ a contraction with coefficient $\alpha(0<\alpha<1)$, and let $A$ be a strongly positive linear bounded operator of $H$ into itself with coefficient $\bar{\gamma}>0$. Assume that $0<\gamma<\bar{\gamma} / \alpha$. Let $\left\{T_{n}\right\}$ be a family of nonexpansive mappings of $H$ into itself, and let $S$ be a $\kappa$-strictly pseudocontraction of $C$ into itself such that

$$
\theta:=\bigcap_{n=1}^{\infty} F\left(T_{n}\right) \cap\left(\bigcap_{k=1}^{N} \operatorname{SMEP}\left(F_{k}\right)\right) \cap F(S) \neq 0 .
$$

Suppose that $\left\{x_{n}\right\}$ is a sequence generated by the following algorithm for $x_{0}, u_{n} \in C$ arbitrarily:

$$
\begin{gathered}
u_{n}=K_{r_{n}, n}^{F_{N}} \cdot K_{r_{n-1}, n}^{F_{N-1}} \cdot K_{r_{n-2}, n}^{F_{N-2}} \cdots K_{r_{2}, n}^{F_{2}} \cdot K_{r_{1}, n}^{F_{1}} \cdot x_{n}, \quad \forall n \in N \\
x_{n+1}=P_{C}\left[\epsilon_{n} \gamma f\left(x_{n}\right)+\left(I-\epsilon_{n} A\right) W_{n}(1-\lambda) x_{n}+\lambda S x_{n}\right]
\end{gathered}
$$

for all $n=0,1,2, \ldots$, and the conditions (C1)-(C3) in Theorem 3.1 are satisfied.

Then, the sequence $\left\{x_{n}\right\}$ converges strongly to $q \in \theta$, where $q=P_{\theta}(\gamma f+I-A)(q)$ which solves the following variational inequality:

$$
\langle(\gamma f-A) q, p-q\rangle \leq 0, \quad \forall p \in \theta
$$

which is the optimality condition for the minimization problem

$$
\min _{q \in \theta} \frac{1}{2}\langle A q, q\rangle-h(q)
$$

where $h$ is a potential function for $\gamma f$ (i.e., $h^{\prime}(q)=\gamma f(q)$ for $\left.q \in H\right)$.

Proof. Put $B \equiv I-T$, then $B$ is $(1-\kappa) / 2$ inverse-strongly monotone and $F(S)=I(B, M)$, and $J_{M, \lambda}\left(x_{n}-\lambda B x_{n}\right)=(1-\lambda) x_{n}+\lambda T x_{n}$. So by Theorem 3.1, we obtain the desired result.

Corollary 4.3. Let $H$ be a real Hilbert space and $C$ a closed convex subset of $H$, and let $B$ be $\beta$-inversestrongly monotone and $\varphi: C \rightarrow R$ a convex and lower semicontinuous function. Let $f: C \rightarrow C$ be a contraction with coefficient $\alpha(0<\alpha<1)$ and $T_{n}$ a nonexpansive mapping of $H$ into itself, and let $S$ be a $\kappa$-strictly pseudocontraction of $C$ into itself such that

$$
\theta:=\bigcap_{n=1}^{\infty} F\left(T_{n}\right) \cap\left(\bigcap_{k=1}^{N} \operatorname{SMEP}\left(F_{k}\right)\right) \cap F(S) \neq 0 .
$$


Suppose that $\left\{x_{n}\right\}$ is a sequence generated by the following algorithm for $x_{0} \in C$ arbitrarily:

$$
\begin{gathered}
u_{n}=K_{r_{n}, n}^{F_{N}} \cdot K_{r_{n-1}, n}^{F_{N-1}} \cdot K_{r_{n-2}, n}^{F_{N-2}} \cdots \cdots K_{r_{2}, n}^{F_{2}} \cdot K_{r_{1}, n}^{F_{1}} \cdot x_{n}, \quad \forall n \in N \\
x_{n+1}=P_{C}\left[\epsilon_{n} f\left(x_{n}\right)+\left(I-\epsilon_{n}\right) W_{n}\left((1-\lambda) u_{n}+\lambda S u_{n}\right)\right]
\end{gathered}
$$

for all $n=0,1,2, \ldots$, and the conditions (C1)-(C3) in Theorem 3.1 are satisfied.

Then, the sequence $\left\{x_{n}\right\}$ converges strongly to $q \in \theta$, where $q=P_{\theta}(f+I)(q)$ which solves the following variational inequality:

$$
\langle(f-I) q, p-q\rangle \leq 0, \quad \forall p \in \theta
$$

which is the optimality condition for the minimization problem

$$
\min _{q \in \theta} \frac{1}{2}\langle A q, q\rangle-h(q)
$$

where $h$ is a potential function for $\gamma f$ (i.e., $h^{\prime}(q)=\gamma f(q)$ for $\left.q \in H\right)$.

Proof. Put $A \equiv I$ and $\gamma \equiv 1$ in Theorem 4.2, we obtain the desired result.

\section{Numerical Example}

Now, we give a real numerical example in which the condition satisfies the ones of Theorem 3.1 and some numerical experiment results to explain the main result Theorem 3.1 as follows.

Example 5.1. Let $H=R, C=[-1,1], T_{n}=I, \lambda_{n}=\beta \in(0,1), n \in N, F_{k}(x, y)=0$, for all $x, y \in C, r_{n, n}=1, k \in\{1,2,3, \ldots, N\}, \varphi(x)=0$, for all $x \in C, B=A=I, f(x)=(1 / 5) x$, for all $x \in H, \lambda=1 / 2$ with contraction coefficient $\alpha=1 / 10, \epsilon_{n}=1 / n$ for every $n \in N$, and $\gamma=1$. Then $\left\{x_{n}\right\}$ is the sequence generated by

$$
x_{n+1}=\left(\frac{1}{2}-\frac{3}{10 n}\right) x_{n}
$$

and $x_{n} \rightarrow 0$ as $n \rightarrow \infty$, where 0 is the unique solution of the minimization problem

$$
\min _{x \in C}=\frac{2}{5} x^{2}+q
$$

Proof. We prove Example 5.1 by Step 1, Step 2, and Step 3. By Step 4, we give two numerical experiment results which can directly explain that the sequence $\left\{x_{n}\right\}$ strongly converges to 0 .

Step 1. We show

$$
K_{r_{n}, n}^{F_{N}} x=P_{C} x, \quad \forall x \in H, F_{N} \in\{1,2,3, \ldots, N\},
$$


where

$$
P_{C} x= \begin{cases}\frac{x}{|x|}, & x \in H \backslash C \\ x, & x \in C .\end{cases}
$$

Indeed, since $F_{k}(x, y)=0$ for all $x, y \in C, n \in\{1,2,3, \ldots, N\}$, due to the definition of $K_{r}(x)$, for all $x \in H$, as Lemma 2.7 , we have

$$
K_{r}(x)=\{u \in C:\langle y-u, u-x\rangle \geq 0, \forall y \in C\} .
$$

Also by the equivalent property (2.2) of the nearest projection $P_{C}$ from $H \rightarrow C$, we obtain this conclusion, when we take $x \in C, K_{r_{n}, n}^{F_{N}} x=P_{C} x=I x$. By (iii) in Lemma 2.7, we have

$$
\bigcap_{k=1}^{N} \operatorname{SMEP}\left(F_{k}\right)=C
$$

Step 2. We show that

$$
W_{n}=I
$$

Indeed. By (1.23), we have

$$
\begin{aligned}
& W_{1}=U_{11}=\lambda_{1} T_{1} U_{12}+\left(1-\lambda_{1}\right) I=\lambda_{1} T_{1}+\left(1-\lambda_{1}\right) I, \\
& \begin{aligned}
W_{2}=U_{21} & =\lambda_{1} T_{1} U_{22}+\left(1-\lambda_{1}\right) I=\lambda_{1} T_{1}\left(\lambda_{2} T_{2} U_{23}+\left(1-\lambda_{2}\right) I\right)+\left(1-\lambda_{1}\right) I \\
& =\lambda_{1} \lambda_{2} T_{1} T_{2}+\lambda_{1}\left(1-\lambda_{2}\right) T_{1}+\left(1-\lambda_{1}\right) I, \\
W_{3}=U_{31} & =\lambda_{1} T_{1} U_{32}+\left(1-\lambda_{1}\right) I=\lambda_{1} T_{1}\left(\lambda_{2} T_{2} U_{33}+\left(1-\lambda_{2}\right) I\right)+\left(1-\lambda_{1}\right) I \\
& =\lambda_{1} \lambda_{2} T_{1} T_{2} U_{33}+\lambda_{1}\left(1-\lambda_{2}\right) T_{1}+\left(1-\lambda_{1}\right) I, \\
& =\lambda_{1} \lambda_{2} T_{1} T_{2}\left(\lambda_{3} T_{3} U_{34}+\left(1-\lambda_{3} I\right)\right)+\lambda_{1}\left(1-\lambda_{2}\right) T_{1}+\left(1-\lambda_{1}\right) I, \\
& =\lambda_{1} \lambda_{2} \lambda_{3} T_{1} T_{2} T_{3}+\lambda_{1} \lambda_{2}\left(1-\lambda_{3}\right) T_{1} T_{2}+\lambda_{1}\left(1-\lambda_{2}\right) T_{1}+\left(1-\lambda_{1}\right) I .
\end{aligned}
\end{aligned}
$$

Computing in this way by (1.23), we obtain

$$
\begin{aligned}
W_{n}=U_{n 1}= & \lambda_{1} \lambda_{2} \cdots \lambda_{n} T_{1} T_{2} \cdots T_{n}+\lambda_{1} \lambda_{2} \cdots \lambda_{n-1}\left(1-\lambda_{n}\right) T_{1} T_{2} \cdots T_{n-1} \\
& +\lambda_{1} \lambda_{2} \cdots \lambda_{n-2}\left(1-\lambda_{n-1}\right) T_{1} T_{2} \cdots T_{n-2}+\cdots+\lambda_{1}\left(1-\lambda_{2}\right) T_{1}+\left(1-\lambda_{1}\right) I .
\end{aligned}
$$

Since $T_{n}=I, \lambda_{n}=\beta, n \in N$, thus

$$
W_{n}=\left[\beta^{n}+\beta^{n-1}(1-\beta)+\cdots+\beta(1-\beta)+(1-\beta)\right] I=I .
$$


Table 1: This table shows the value of sequence $\left\{x_{n}\right\}$ on each iteration step (initial value $x_{1}=1$ ).

\begin{tabular}{lccc}
\hline$n$ & $x_{n}$ & $n$ & $x_{n}$ \\
\hline 1 & 1.000000000000000 & 31 & 0.000000000054337 \\
2 & 0.200000000000000 & 32 & 0.000000000026643 \\
3 & 0.070000000000000 & 33 & 0.000000000013072 \\
4 & 0.028000000000000 & 34 & 0.000000000006417 \\
$\vdots$ & $\vdots$ & $\vdots$ & $\vdots$ \\
19 & 0.000000301580666 & 39 & 0.000000000000184 \\
20 & 0.000000146028533 & 40 & 0.000000000000091 \\
21 & 0.000000070823839 & 41 & 0.000000000000045 \\
$\vdots$ & $\vdots$ & $\vdots$ & $\vdots$ \\
29 & 0.000000000226469 & 47 & 0.000000000000001 \\
30 & 0.000000000110892 & 48 & 0.000000000000000 \\
\hline
\end{tabular}

Step 3. We show that

$$
x_{n+1}=\left(\frac{1}{2}-\frac{3}{10 n}\right) x_{n}, \quad x_{n+1} \longrightarrow 0, \text { as } n \longrightarrow \infty,
$$

where 0 is the unique solution of the minimization problem

$$
\min _{x \in C} \frac{2}{5} x^{2}+q
$$

Indeed, we can see that $A=I$ is a strongly position bounded linear operator with coefficient $\bar{\gamma}=1 / 2$ and $\gamma$ is a real number such that $0<\gamma<\bar{\gamma} / \alpha$, so we can take $\gamma=1$. Due to (5.1), (5.4), and (5.7), we can obtain a special sequence $\left\{x_{n}\right\}$ of (3.2) in Theorem 3.1 as follows:

$$
x_{n+1}=\left(\frac{1}{2}-\frac{3}{10 n}\right) x_{n}
$$

Since $T_{n}=I, n \in N$, so,

$$
\bigcap_{n=1}^{\infty} F\left(T_{n}\right)=H
$$

combining with (5.6), we have

$$
\theta:=\bigcap_{n=1}^{\infty} F\left(T_{n}\right) \cap\left(\bigcap_{k=1}^{N} \operatorname{SMEP}\left(F_{k}\right)\right) \cap I(B, M)=C=[-1,1]
$$


Table 2: This table shows the value of sequence $\left\{x_{n}\right\}$ on each iteration step (initial value $x_{1}=1 / 2$ ).

\begin{tabular}{lccc}
\hline$n$ & $x_{n}$ & $n$ & $x_{n}$ \\
\hline 1 & 0.500000000000000 & 31 & 0.000000000027168 \\
2 & 0.100000000000000 & 32 & 0.000000000013321 \\
3 & 0.035000000000000 & 33 & 0.000000000006536 \\
4 & 0.014000000000000 & 34 & 0.000000000003208 \\
$\vdots$ & $\vdots$ & $\vdots$ & $\vdots$ \\
19 & 0.000000150790333 & 39 & 0.000000000000092 \\
20 & 0.000000073014267 & 40 & 0.000000000000045 \\
21 & 0.000000035411919 & 41 & 0.000000000000022 \\
$\vdots$ & $\vdots$ & $\vdots$ & $\vdots$ \\
29 & 0.000000000113235 & 46 & 0.000000000000001 \\
30 & 0.000000000055446 & 47 & 0.000000000000000 \\
\hline
\end{tabular}

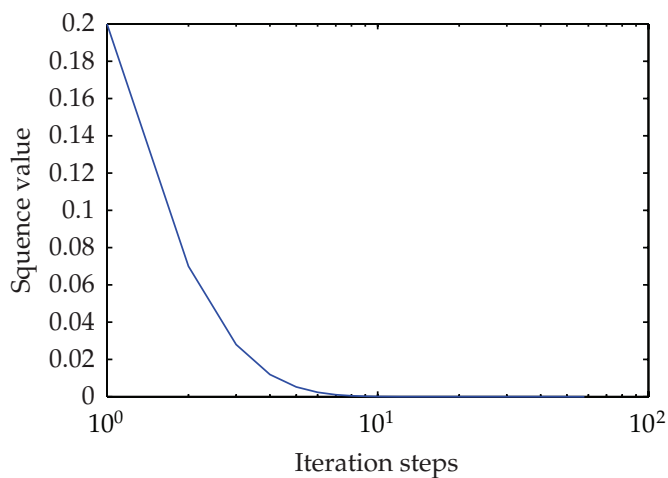

(a)

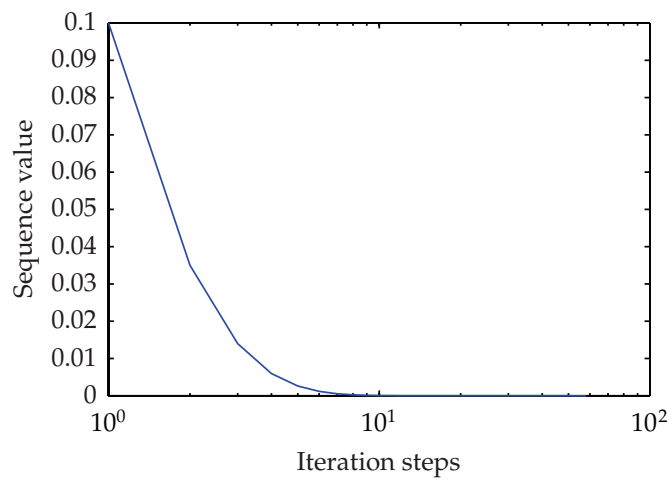

(b)

Figure 1: The iteration comparison chart of different initial values. (a) $x_{1}=1$ and (b) $x_{1}=1 / 2$. problem

By Lemma 2.5, it is obviously that $z_{n} \rightarrow 0,0$ is the unique solution of the minimization

$$
\min _{x \in C} \frac{2}{5} x^{2}+q
$$

where $q$ is a constant number.

Step 4. We give the numerical experiment results using software Mathlab 7.0 and get Table 1 to Table 2, which show that the iteration process of the sequence $\left\{x_{n}\right\}$ is a monotonedecreasing sequence and converges to 0 , but the more the iteration steps are, the more showily the sequence $\left\{x_{n}\right\}$ converges to 0 .

Now, we turn to realizing (3.2) for approximating a fixed point of $T$. We take the initial valued $x_{1}=1$ and $x_{1}=1 / 2$, respectively. All the numerical results are given in Tables 1 and 2. The corresponding graph appears in Figures 1(a) and 1(b). 
The numerical results support our main theorem as shown by calculating and plotting graphs using Matlab 7.11.0.

\section{Acknowledgments}

The authors would like to thank the Higher Education Research Promotion and National Research University Project of Thailand, Office of the Higher Education Commission (under the project NRU-CSEC no. 54000267) for financial support. Furthermore, the second author was supported by the Commission on Higher Education, the Thailand Research Fund, and the King Mongkut's University of Technology Thonburi (KMUTT) (Grant no. MRG5360044). Finally, the authors would like to thank the referees for reading this paper carefully, providing valuable suggestions and comments, and pointing out major errors in the original version of this paper.

\section{References}

[1] E. Blum and W. Oettli, "From optimization and variational inequalities to equilibrium problems," The Mathematics Student, vol. 63, no. 1-4, pp. 123-145, 1994.

[2] P. L. Combettes and S. A. Hirstoaga, "Equilibrium programming in Hilbert spaces," Journal of Nonlinear and Convex Analysis, vol. 6, no. 1, pp. 117-136, 2005.

[3] S. D. Flåm and A. S. Antipin, "Equilibrium programming using proximal-like algorithms," Mathematical Programming, vol. 78, no. 1, pp. 29-41, 1997.

[4] S. Takahashi and W. Takahashi, "Viscosity approximation methods for equilibrium problems and fixed point problems in Hilbert spaces," Journal of Mathematical Analysis and Applications, vol. 331, no. 1, pp. 506-515, 2007.

[5] T. Jitpeera, U. Witthayarat, and P. Kumam, "Hybrid algorithms of common solutions of generalized mixed equilibrium problems and the common variational inequality problems with applications," Fixed Point Theory and Applications, vol. 2011, Article ID 971479, 28 pages, 2011.

[6] T. Jitpeera and P. Kumam, "An extragradient type method for a system of equilibrium problems, variational inequality problems and fixed points of finitely many nonexpansive mappings," Journal of Nonlinear Analysis and Optimization: Theory E Applications, vol. 1, no. 1, pp. 71-91, 2010.

[7] T. Jitpeera and P. Kumam, "A new hybrid algorithm for a system of equilibrium problems and variational inclusion," Annali dell'Universita di Ferrara, vol. 57, no. 1, pp. 89-108, 2011.

[8] T. Jitpeera and P. Kumam, "Hybrid algorithms for minimization problems over the solutions of generalized mixed equilibrium and variational inclusion problems," Mathematical Problems in Engineering, vol. 2011, Article ID 648617, 25 pages, 2011.

[9] P. Kumam, U. Hamphries, and P. Katchang, "Common solutions of generalized mixed equilibrium problems, variational inclusions and common fixed points for nonexpansive semigroups and strictly pseudo-contractive mappings," Journal of Applied Mathematics, vol. 2011, Article ID 953903, 27 pages, 2011.

[10] P. Sunthrayuth and P. Kumam, "A new general iterative method for solution of a new general system of variational inclusions for nonexpansive semigroups in Banach spaces," Journal of Applied Mathematics, vol. 2011, Article ID 187052, 29 pages, 2011.

[11] P. Katchang and P. Kumam, "Convergence of iterative algorithm for finding common solution of fixed points and general system of variational inequalities for two accretive operators," Thai Journal of Mathematics, vol. 9, no. 2, pp. 319-335, 2011.

[12] W. Kumam, P. Junlouchai, and P. Kumam, "Generalized systems of variational inequalities and projection methods for inverse-strongly monotone mappings," Discrete Dynamics in Nature and Society, vol. 2011, Article ID 976505, 24 pages, 2011.

[13] P. Kumam, "A relaxed extragradient approximation method of two inverse-strongly monotone mappings for a general system of variational inequalities, fixed point and equilibrium problems," Bulletin of the Iranian Mathematical Society, vol. 36, no. 1, pp. 227-250, 2010. 
[14] P. Kumam and C. Jaiboon, "Approximation of common solutions to system of mixed equilibrium problems, variational inequality problem, and strict pseudo-contractive mappings," Fixed Point Theory and Applications, vol. 2011, Article ID 347204, 30 pages, 2011.

[15] C. Jaiboon and P. Kumam, "A general iterative method for addressing mixed equilibrium problems and optimization problems," Nonlinear Analysis: Theory, Methods \& Applications A, vol. 73, no. 5, pp. 1180-1202, 2010.

[16] Y. J. Cho, I. K. Argyros, and N. Petrot, "Approximation methods for common solutions of generalized equilibrium, systems of nonlinear variational inequalities and fixed point problems," Computers $\mathcal{E}$ Mathematics with Applications, vol. 60, no. 8, pp. 2292-2301, 2010.

[17] Y. J. Cho, N. Petrot, and S. Suantai, "Fixed point theorems for nonexpansive mappings with applications to generalized equilibrium and system of nonlinear variational inequalities problems," Journal of Nonlinear Analysis and Optimization, vol. 1, no. 1, pp. 45-53, 2010.

[18] Y. J. Cho and N. Petrot, "On the system of nonlinear mixed implicit equilibrium problems in Hilbert spaces," Journal of Inequalities and Applications, vol. 2010, Article ID 437976, 12 pages, 2010.

[19] Y. Yao, Y. J. Cho, and Y.-C. Liou, "Algorithms of common solutions for variational inclusions, mixed equilibrium problems and fixed point problems," European Journal of Operational Research, vol. 212, no. 2, pp. 242-250, 2011.

[20] W. A. Kirk, "Fixed point theorem for mappings which do not increase distances," The American Mathematical Monthly, vol. 72, pp. 1004-1006, 1965.

[21] P. Hartman and G. Stampacchia, "On some non-linear elliptic differential-functional equations," Acta Mathematica, vol. 115, pp. 271-310, 1966.

[22] J.-C. Yao and O. Chadli, "Pseudomonotone complementarity problems and variational inequalities," in Handbook of Generalized Convexity and Monotonicity, J. P. Crouzeix, N. Haddjissas, and S. Schaible, Eds., vol. 76, pp. 501-558, Springer, New York, NY, USA, 2005.

[23] Y. Yao and N. Shahzad, "New methods with perturbations for non-expansive mappings in Hilbert spaces," Fixed Point Theory and Applications, vol. 2011, article 79, 2011.

[24] Y. Yao and N. Shahzad, "Strong convergence of a proximal point algorithm with general errors," European Journal of Operational Research. In Press.

[25] Y. Yao, Y.-C. Liou, and C.-P. Chen, "Algorithms construction for nonexpansive mappings and inversestrongly monotone mappings," Taiwanese Journal of Mathematics, vol. 15, no. 5, pp. 1979-1998, 2011.

[26] Y. Yao, R. Chen, and Y.-C. Liou, "A unified implicit algorithm for solving the triple-hierarchical constrained optimization problem," Mathematical and Computer Modelling, vol. 55, pp. 1506-1515, 2012.

[27] Y. Yao, Y.-C. Liou, S. M. Kang, and Y. Yu, "Algorithms with strong convergence for a system of nonlinear variational inequalities in Banach spaces," Nonlinear Analysis, Theory, Methods and Applications, vol. 74, no. 17, pp. 6024-6034, 2011.

[28] Y. Yao, Y.-C. Liou, and S. M. Kang, "Two-step projection methods for a system of variational inequality problems in Banach spaces," Journal of Global Optimization. In Press.

[29] G. Marino and H.-K. Xu, "A general iterative method for nonexpansive mappings in Hilbert spaces," Journal of Mathematical Analysis and Applications, vol. 318, no. 1, pp. 43-52, 2006.

[30] Y. Hao, "Some results of variational inclusion problems and fixed point problems with applications," Applied Mathematics and Mechanics, vol. 30, no. 12, pp. 1589-1596, 2009.

[31] M. Liu, S. S. Chang, and P. Zuo, “An algorithm for finding a common solution for a system of mixed equilibrium problem, quasivariational inclusion problem, and fixed point problem of nonexpansive semigroup," Journal of Inequalities and Applications, vol. 2010, Article ID 895907, 23 pages, 2010.

[32] S.-s. Zhang, J. H. W. Lee, and C. K. Chan, "Algorithms of common solutions to quasi variational inclusion and fixed point problems," Applied Mathematics and Mechanics, vol. 29, no. 5, pp. 571-581, 2008.

[33] R. T. Rockafellar, "On the maximality of sums of nonlinear monotone operators," Transactions of the American Mathematical Society, vol. 149, pp. 46-55, 2000.

[34] B. Lemaire, "Which fixed point does the iteration method select?" in Recent Advances in Optimization, vol. 452 of Lecture Note in Economics and Mathematical Systems, pp. 154-167, Springer, Berlin, Germany, 1997.

[35] A. Moudafi, "Viscosity approximation methods for fixed-points problems," Journal of Mathematical Analysis and Applications, vol. 241, no. 1, pp. 46-55, 2000.

[36] H. Iiduka and W. Takahashi, "Strong convergence theorems for nonexpansive mappings and inversestrongly monotone mappings," Nonlinear Analysis: Theory, Methods \& Applications A, vol. 61, no. 3, pp. 341-350, 2005. 
[37] Y. Su, M. Shang, and X. Qin, "An iterative method of solution for equilibrium and optimization problems," Nonlinear Analysis: Theory, Methods E Applications A, vol. 69, no. 8, pp. 2709-2719, 2008.

[38] H. He, S. Liu, and Y. J. Cho, "An explicit method for systems of equilibrium problems and fixed points of infinite family of nonexpansive mappings," Journal of Computational and Applied Mathematics, vol. 235, no. 14, pp. 4128-4139, 2011.

[39] T. Jitpeera and P. Kumam, “A general iterative algorithm for generalized mixed equilibrium problems and variational inclusions approach to variational inequalities," International Journal of Mathematics and Mathematical Sciences, Article ID 619813, 25 pages, 2011.

[40] H. Brézis, "Opérateur maximaux monotones," in Mathematics Studies, vol. 5, North-Holland, Amsterdam, The Netherlands, 1973.

[41] Z. Opial, "Weak convergence of the sequence of successive approximations for nonexpansive mappings," Bulletin of the American Mathematical Society, vol. 73, pp. 591-597, 1967.

[42] H.-K. Xu, "Iterative algorithms for nonlinear operators," Journal of the London Mathematical Society, vol. 66, no. 1, pp. 240-256, 2002.

[43] F. E. Browder, Ed., Nonlinear Operators and Nonlinear Equations of Evolution in Banach Spaces, vol. 18 of Proceedings of Symposia in Pure Mathematics, American Mathematical Society, 1976.

[44] J.-W. Peng, Y.-C. Liou, and J.-C. Yao, “An iterative algorithm combining viscosity method with parallel method for a generalized equilibrium problem and strict pseudocontractions," Fixed Point Theory and Applications, vol. 2009, Article ID 794178, 21 pages, 2009.

[45] C. Klin-Eam and S. Suantai, "A new approximation method for solving variational inequalities and fixed points of nonexpansive mappings," Journal of Inequalities and Applications, vol. 2009, Article ID 520301, 16 pages, 2009. 


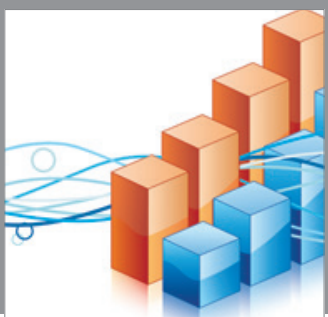

Advances in

Operations Research

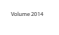

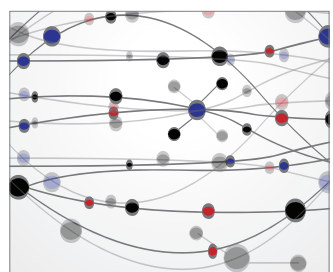

\section{The Scientific} World Journal
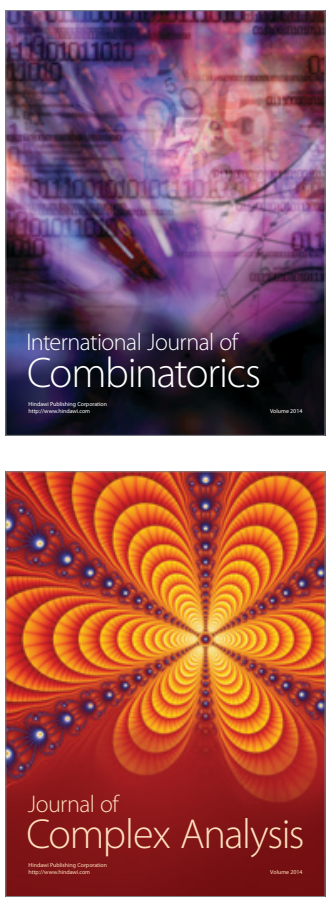

International Journal of

Mathematics and

Mathematical

Sciences
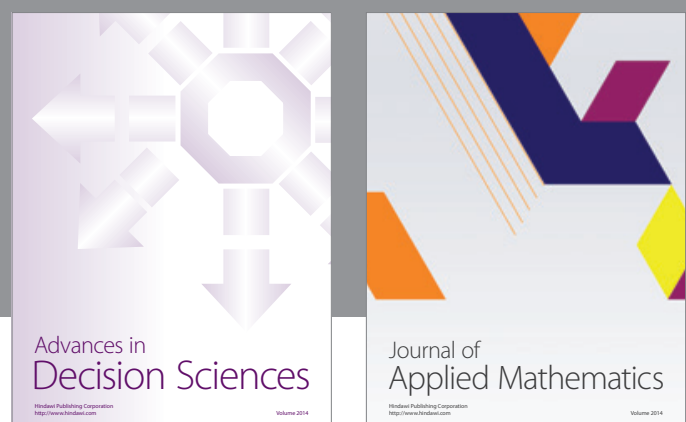

Journal of

Applied Mathematics
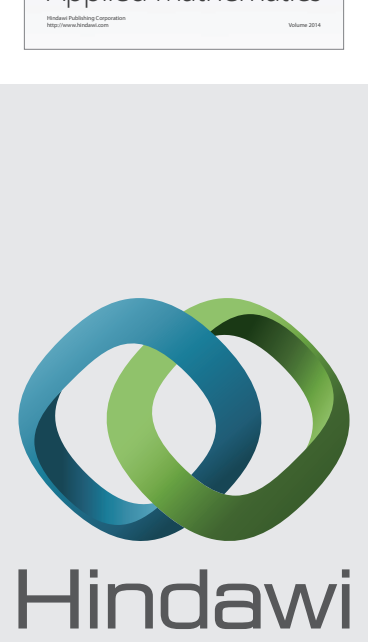

Submit your manuscripts at http://www.hindawi.com
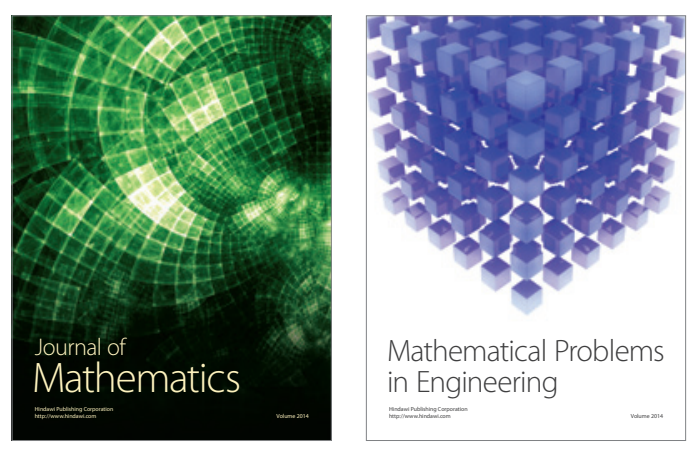

Mathematical Problems in Engineering
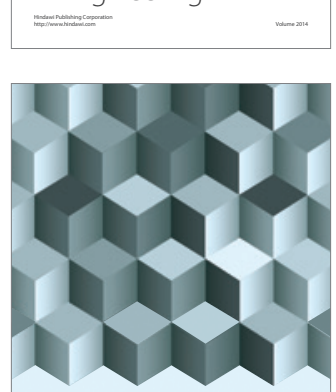

Journal of

Function Spaces
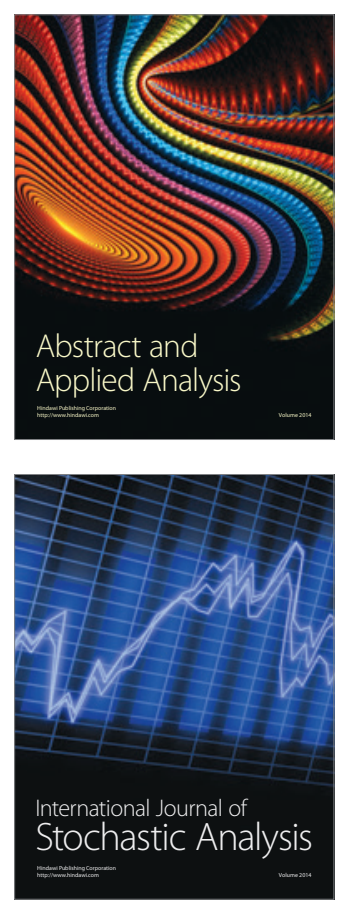

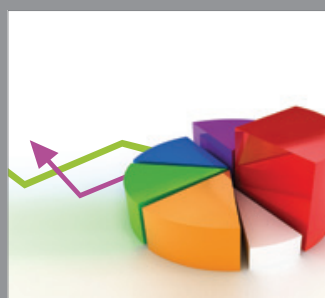

ournal of

Probability and Statistics

Promensencen
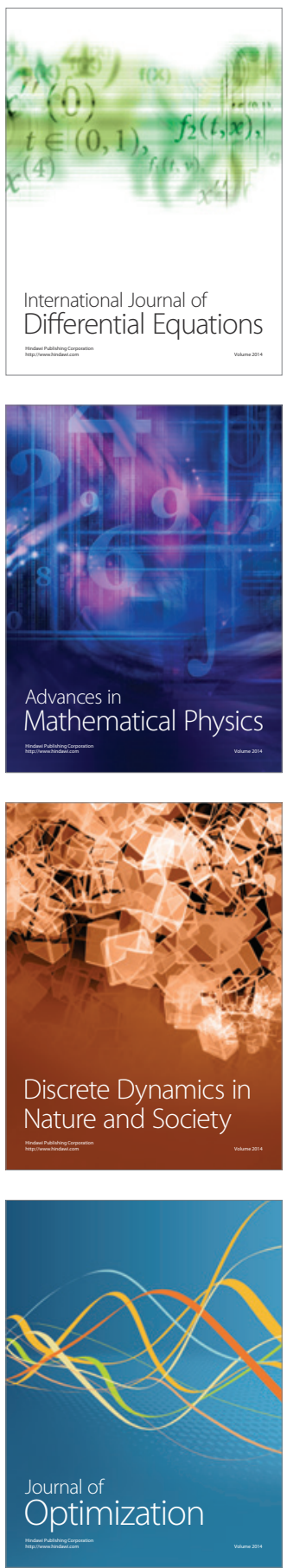